\title{
Extended continuum configurational bias Monte Carlo methods for simulation of flexible molecules
}

\author{
Fernando A. Escobedo and Juan J. de Pablo \\ Department of Chemical Engineering, University of Wisconsin, Madison, Wisconsin 53706
}

(Received 25 August 1994; accepted 3 November 1994)

\begin{abstract}
The continuum configurational bias (CCB) Monte Carlo method has been extended to perform elementary moves that involve the rearrangement of inner segments of flexible chains. When regrowing inner sites, the continuity with the rest of the chain is ensured by disregarding those configurations that would imply an unrealistic elongation of the bonds once the chain is reconstructed. The formalism presented here also allows the simulation of branched chains and crosslinked-network structures. The Monte Carlo elementary moves proposed in this work are used in conjunction with an alternative method of preferential sampling in which the segments to be rearranged are chosen from a preselected region of space. The performance and capabilities of the new moves are compared to those of standard CCB and crank-shaft algorithms for simulation of melts and solutions of hard-sphere chains at high densities. Our results indicate that the methods presented here provide a fast relaxation of the bond orientation and the end-to-end orientation autocorrelation functions. Our isobaric simulations for homopolymer chains of up to 51 sites and for concentrated solutions of chain molecules in the monomer are consistent with previously reported data obtained by approximate molecular dynamics methods and by conventional Monte Carlo methods. However, small disagreements with existing data are identified at high densities. These PV results are also compared to the predictions of two recent equations of state. This comparison shows the presence of some small but systematic deviations. (C) 1995 American Institute of Physics.
\end{abstract}

\section{INTRODUCTION}

Much of our current understanding of simple fluids has emerged from molecular simulations. In recent years more complex, molecular fluids have received increasing amounts of attention. Polymers, in particular, have been studied by computer simulations by a number of authors. ${ }^{1-4}$ More often than not, such studies have resorted to methods that were originally designed for simple fluids and that are ill-suited for long chain molecules. Improved techniques for simulation of polymeric systems have begun to appear only recently, ${ }^{5-8}$ and there are still many problems that need to be addressed. ${ }^{8,9}$

Computer simulations have played an increasingly important role in polymer physics by providing "exact" results for the structure and properties of model systems. ${ }^{10}$ In that context, the study of hard-sphere chains is a natural starting point for theoretical work because the structure of liquids is largely determined by short-range, repulsive forces. In spite of their importance, however, simulations of hard-sphere chain have been limited to moderate densities and short chain lengths. Simulations in the canonical ensemble have been reported for chains of up to 100 beads and packing fractions $(\eta)$ of up to $0.30 ;{ }^{11}$ but, to the best of our knowledge, study of their PV behavior has been restricted to chains of up to 16 segments at $\eta \leqslant 0.376 .{ }^{12-14}$ Difficulties associated with simulation of longer chains at high densities have precluded more complete and extensive studies.

In recent years several equations of state for hard-sphere chain molecules have been proposed. ${ }^{12,15-22}$ Such equations constitute the backbone for almost any theory of condensed polymers. The relative merits of available models, however, have only been discussed on the grounds of limited and sometimes inaccurate simulation data. The generation of PV data for a more comprehensive variety of hard-sphere chain systems and over a wider range of conditions would enable the development of more accurate equations of state, not only for hard-body systems but also for realistic polymeric systems.

It is difficult to simulate hard-sphere chains by conventional molecular dynamics methods; ${ }^{23}$ the complexity of the required collision dynamics has eluded their practical implementation. It should be noted that Gao and Weiner ${ }^{24}$ have been able to use molecular dynamics to simulate hard chains by approximating hard-core interactions by a soft, LennardJones type of potential-energy function. Unfortunately, a conclusive and independent means of assessing the validity and accuracy of their approximate results at high densities is not available. Furthermore, their approach would be difficult to apply to heteronuclear chains and mixtures.

It is therefore necessary to develop new Monte Carlo methods that are capable of exploring the configuration space of such systems in a manner that is sufficiently efficient to provide reliable estimates of their equilibrium properties. Monte Carlo methods are particularly suitable for simulation of hard-core systems because the fundamental restriction to any given configuration of the system is simply the avoidance of overlaps. ${ }^{23}$ Incidentally, this feature makes hard-sphere-chain systems ideal for development and testing of new Monte Carlo algorithms; since the complexity of intramolecular and intermolecular interactions is minimal, the performance of a Monte Carlo move will depend only on the structure of the system and the simulation algorithm itself.

In recent years, Monte Carlo methods for simulation of polymeric systems have experienced significant advances. Most of these methods have been used to investigate lattice 
systems. ${ }^{3-5,25}$ In this work, however, we are interested in the study of polymers in a continuum; for conciseness, we make little reference to studies on a lattice.

Off-lattice Monte Carlo simulations of polymers have been limited. The reptation algorithm ${ }^{1,2,26,27}$ was probably the first prescription that substantially improved the performance of conventional Metropolis Monte Carlo moves for simulation of chain molecules. Recently, a more powerful technique came along with the development of the continuum configurational bias Monte Carlo method ${ }^{6}$ (CCB); by cutting and regrowing many sites at one end of the chain, the CCB method can achieve drastic conformational rearrangements and better sampling of the configurational space. Unfortunately, the efficiency of both reptation and CCB methods decreases significantly as the chain becomes longer. ${ }^{9}$ The main reason for such a failure is that both of these methods operate at or near the ends of the chains; for long molecules, the concentration of chain ends is often low.

Reptation and CCB rely on the occurrence of empty spaces for the relocation of monomeric units of the chains. At high densities, void spaces are rare, thereby reducing even further the efficiency of these methods. ${ }^{9}$ The various shortcomings and advantages of different simulation algorithms have been discussed at great length in earlier publications ${ }^{6,7,9}$ to which the reader is referred for further details.

Reptation and CCB moves are equally applicable to fully or partially flexible chains. For molecules with rigid constraints (i.e., fixed bond lengths and rigid bond angles), such as alkanelike architectures, some of the shortcomings of such moves can be alleviated by concerted rotations, which achieve a localized elementary move of a few consecutive sites along the backbone of a chain. ${ }^{8,9}$ The performance of this method is basically chain-length independent and appears to work reasonably well at high densities. Unfortunately, the advantages of concerted rotations are offset by the expense of programming and solving a relatively complicated system of equations entailed by the geometric constraints of the molecule. It should be stressed that concerted rotations are only applicable to molecules with rigid constraints. Recently, CCB and concerted-rotation moves have been combined with some benefit for simulation of long alkanes. ${ }^{9,28}$ The advantages of such a combined algorithm, however, are not dramatic.

For simulation of fully flexible chains, researchers have also implemented several variants of what could be loosely called a "random" move. In one example, a small translation of the whole molecule is accompanied by small changes in the direction of each bond along the chain. ${ }^{11,15}$ Such "random" moves tend to be inefficient and are not particularly useful for simulation of long chains.

A crank-shaft type of move has also been used for simulation of flexible chains. ${ }^{29,30}$ Such a move consists of rotating individual sites, one at a time; the performance of crankshaft algorithms is essentially chain-length independent, thereby leading to a robust simulation method. Crank-shaft moves have been implemented in a continuum by direct extension of a prescription that was originally devised for lattice systems. ${ }^{31}$ However, it has not been shown that crankshaft moves satisfy microscopic reversibility when applied to off-lattice models. Furthermore, to the best of our knowledge, these moves have not been fully tested at high densities, where they might have only a limited success.

Copolymers consisting of alternating but distinct monomers are important in practice. Reptation methods are of little use for their simulation because they violate structural constraints. Branched and crosslinked polymers are also of great practical importance. None of the conventional methods mentioned so far are well suited to simulate these systems. Such materials therefore represent challenging systems on which to perform Monte Carlo simulations. Clearly, a simulation method that is equally applicable to simple homopolymer chains, branched chains, crosslinked polymers or heteropolymer chains would be desirable.

In this work we present a new Monte Carlo method for simulation of flexible chains. It is equally applicable to homopolymers, copolymers, and branched polymers. Its versatility permits the simultaneous displacement of multiple sites, thereby increasing the chances for cooperative rearrangements of the molecules. Such rearrangements can be made more localized by implementing a preferential sampling in confined regions of space (HOLE method) where the moves are constrained to occur.

In the next section we summarize the theoretical basis for the new moves proposed in this work. In Sec. III we present some tests to validate the new moves. A description of the computational methods and performance criteria adopted in our work is given in Sec. IV. The application of these methods to the simulation of hard-sphere chains in the NVT and NPT ensembles is given and discussed in Sec. V. Finally, in Sec. VI we provide some concluding remarks and suggestions for future work.

\section{THEORY}

Our discussion will focus on methods capable of dealing with flexible chain molecules (or semiflexible chains modeled by including a bending potential energy between contiguous bonds). Each chain is modeled as a sequence of sites linked by rigid bonds ("Kramers" pearl-necklace model ${ }^{32}$ ). Each site or bead may represent an atom or a group of atoms, depending on the specific system to be modeled. Noncontiguous sites or sites belonging to different chains may interact with any potential energy function as long as the sites can be characterized by a "hard-sphere" diameter, i.e., some minimum distance that imposes an energy barrier for the penetration by any other site. Furthermore, the sites may have different sizes and may be connected by rigid bonds of different lengths.

\section{A. The CCB method}

The basis of this method was first developed in lattice models. ${ }^{5,33-36}$ It was then extended to a continuum by de Pablo et al. ${ }^{6}$ and by Frenkel et al. ${ }^{37}$ The capabilities and limitations of the method have been addressed in various studies ${ }^{7,9,37}$ and need not be discussed again. Here it suffices to describe the elementary steps that constitute the method.

A randomly selected chain is cut at some random point. A portion of the chain from this point to one of the ends is deleted. 
The chain is regrown site by site to its original length (Fig. 1). For each site to be regrown, $N_{\text {samp }}$ trial orientations of the connecting bond vector are generated. One of them is chosen with probability: ${ }^{6}$

$$
\begin{aligned}
w_{i} & =\exp \left(-\beta U_{i}\right) / \sum_{k=1}^{N_{\text {samp }}} \exp \left(-\beta U_{k}\right) \\
& =w_{i}^{*} / \sum_{k=1}^{N_{\text {samp }}} w_{k}^{*},
\end{aligned}
$$

where $w_{i}$ is the normalized Boltzmann factor for the $i$ th trial configuration with energy $U_{i}\left(w_{i}^{*}\right.$ for the non-normalized weight) and $\beta=\left(k_{B} T\right)^{-1}$ ( $k_{B}$ is Boltzmann's constant).

This way of selecting the new site position introduces a bias that must be removed to ensure microscopic reversibility. This is done by introducing the weights $w_{i}$ into the acceptance criteria of the proposed move according to ${ }^{6}$

$$
P_{\mathrm{CCB}}=\min \left(1, \frac{W_{\text {chain }}^{\text {old }} P_{\text {new }}}{W_{\text {chain }}^{\text {new }} P_{\text {old }}}\right),
$$

where

$$
W_{\text {chain }}=\prod_{i=1}^{N_{\text {sites }}} w_{i}
$$

and where $P_{\text {new }} / P_{\text {old }}$ is the ratio of the probabilities of the new and old configurations in the system. For flexible chains, the possible positions of each appended site are sought uniformly on a spherical surface centered at the previous existing site.

\section{B. Implementation of the crank-shaft move}

Various implementations of crank-shaft moves have been used for lattice simulations. ${ }^{4,31} \mathrm{~A}$ one-site move can be easily extended to off-lattice simulations of flexible chains and has been used for the simulation of polymer melts by various authors. ${ }^{29,30}$ The move consists of choosing an inner site of the chain and then rotating it around the axis formed by the line that joins the centers of the two adjacent sites (see Fig. 1). In principle, the extent of the rotation should be chosen at random. A more refined application of the method should introduce a means of controlling the acceptance rate of the attempted moves. There are a number of ways for doing so.

One possibility is to add to the "old" position of the rotating vector another randomly oriented vector located on the same plane of rotation. The acceptance rate can be controlled by adjusting the magnitude of the second vector. An important disadvantage of this or other related methods is that at high densities, when moves are unlikely to succeed, the magnitude of the modifying vector must be small if a reasonable acceptance rate is to be maintained. This restricts the moves to small rotations only, thereby leading to an inefficient way of producing conformational changes.

This problem can be circumvented by implementing crank-shaft moves in a CCB fashion; instead of attempting one random rotation of a given site, several random rotations

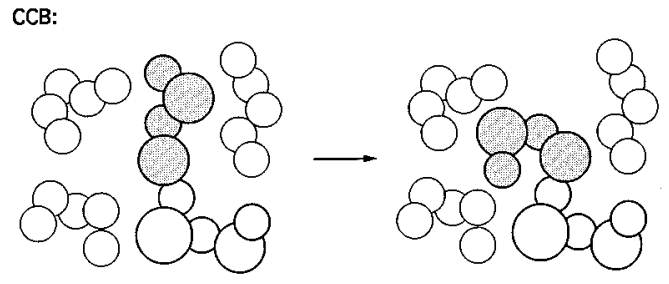

CRANK-SHAFT:

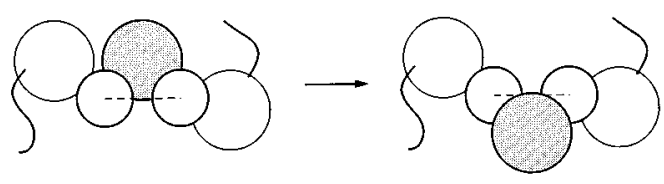

FIG. 1. Illustration of a standard CCB move and a crank-shaft move.

are sampled over the whole rotational circumference. One of these positions is then chosen according to Eq. (1).

It is important to note that in any implementation of a crank-shaft move, there is a local change of the configurational space variables (i.e., the degrees of freedom used to determine the new position of the moved site); the Jacobian determinant $J$ associated with such a transformation of coordinates must be included in the acceptance criteria ${ }^{8,9}$ according to

$$
P_{\mathrm{CCB}}=\min \left(1, \frac{J^{\text {new }}}{J^{\text {old }}} \frac{W_{\text {chain }}^{\text {old }} P_{\text {new }}}{W_{\text {chain }}^{\text {new }} P_{\text {old }}}\right) .
$$

This Jacobian has been ignored by most authors; in Appendix A we show that, fortunately, the ratio of the Jacobian determinants in this equation is always equal to one. As shown later, crank-shaft moves implemented using configurational bias ideas can be more efficient than conventional, "brute-force" crank-shaft moves, particularly at conditions for which the acceptance rate of the latter method becomes small.

\section{The new move (ECCB)}

A critical shortcoming of the original CCB move is that only end sections of the chain can be regrown. This renders the method inefficient for long chains, where only the outmost sites of a chain move significantly. As reported for lattice models, ${ }^{25}$ a method that modifies the inner structure of a chain would have significant advantages.

The method proposed in this section consists of erasing an arbitrary section of a chain molecule, and regrowing it, segment by segment, until the original chain length of the molecule is restored. If inner sites of the chains are to be cut, it is necessary to devise some means to ensure that the regrown sites properly "close" the chain. To satisfy such geometric constraints in an efficient manner, a systematic check of the physical realizability of a configuration should be performed in advance, every time that a site is appended to the growing molecule, and not only at the time of closing the chain. This can be done by identifying an "available" sam- 


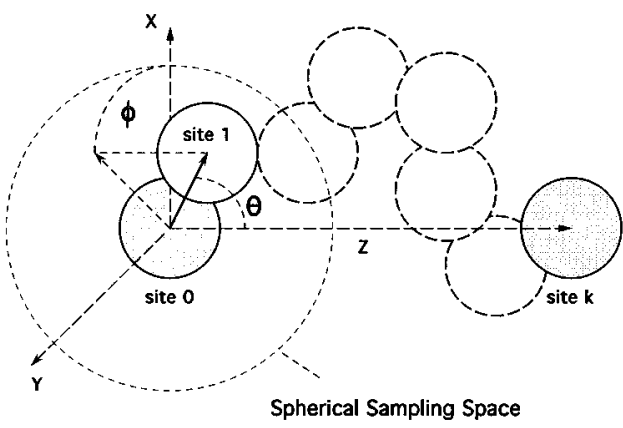

FIG. 2. Nomenclature and local coordinate system for the ECCB move. Sites 0 and $k$ are the open ends of the chain and 1 is the growing site.

pling space for the direction of each bond that is appended. In a conventional $\mathrm{CCB}$ move, the direction of the new bond vector is randomly chosen on the whole surface of a sphere. For inner segments, the proposed ECCB move requires that the new bond vector be sampled on just a fraction of the spherical surface that would otherwise be available to it.

Figure 2 defines the coordinate system used in this work; we specify the boundaries of such a surface by imposing bounds to the spherical angle $\theta$ formed by the bond vector and the $z$ axis along the line connecting the centers of the previous pivotal site and the linking site at the other side of the chain (sites 0 and $k$ in Fig. 2, respectively). These bounds are completely defined once the position of the nearest two "existing" sites at each side of the cut are specified. These sites are the last appended site at one side of the chain, and the first uncut site at the other side of the chain. This fact is illustrated in Fig. 3 for the case where two inner sites (1 and 2) of a hard-sphere chain are to be relocated. Figure 3(a) illustrates an extreme case where spheres 0 and 3 are at their maximum separation, i.e., the centers of the four sites $0,1,2$,

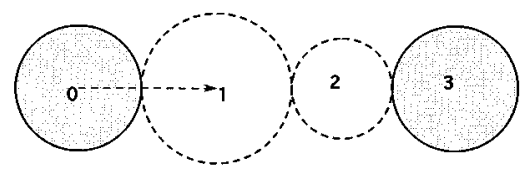

(a)

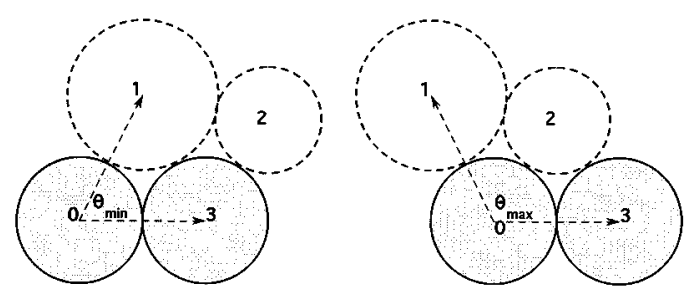

(b)

(c)

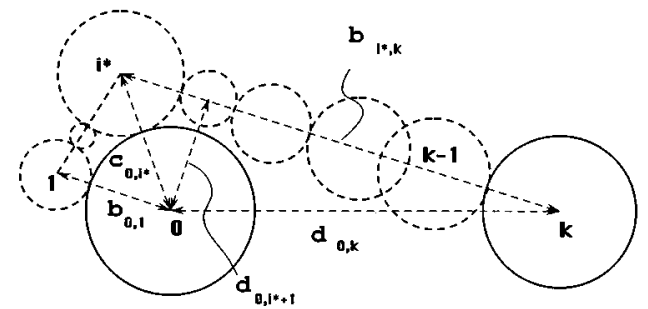

FIG. 4. Nomenclature used to determine the maximal extension of intermediate segments.

and 3 lie on a straight line. Clearly, if site 1 is to be appended next to site 0 , it can only take one position, i.e., its sampling space is reduced to a point. On the other hand, Figs. 3(b) and 3(c) show cases where the "existing" sites 0 and 3 are at their closest approach. In that event, site 1 has very distinctive lower and upper bounds $\theta_{\min }$ and $\theta_{\max }$. Note, however, that there are no restrictions on the spherical angle $\phi$ to be chosen when sampling the orientation of a bond vector (see Fig. 2).

For the general case where $N_{\text {cut }}$ sites are cut and regrown, the first site to be appended will have the greater sampling surface available to it. This surface will be subsequently reduced as an increasing number of sites is appended to the chain. For the last site to be inserted, the sampling surface is reduced to a circumference, thereby resulting in a simple crank-shaft move.

In principle, the regrowing process can take place from one side of the growing section to the other, with direction chosen at random, or it can take place in alternating directions, site by site, in a regular or random sequence. In this study only the first approach has been implemented.

We now propose a solution to the geometric problem entailed by the determination of the bounds of the $\theta$ angle. The nomenclature to be used is illustrated in Fig. $4 ; d_{i, j}$ denotes the straight line distance between the centers of sites $i$ and $j$ in any given configuration, $b_{i, j}$ denotes the distance along the connecting bonds from site $i$ to site $j$, if all intermediate sites were aligned on a straight line with sites $i$ and $j$, and $c_{i, j}$ is the shortest possible distance between sites $i$ and $j$, i.e., $c_{i, j}=\left(r_{i}+r_{j}\right)$, where $r_{i}$ is radius of site $i$. Note that $c_{i, j}=b_{i, j}$ only if $j$ is next to $i$ on the chain (i.e., only if $j=i \pm 1$ ). As before, site 0 is the "pivot" site to which the growing site 1 is to be appended. Sites 1 to $k-1$ remain to be regrown.

The specification of the lower bound, $\theta_{\min }$, is not relevant to ensure the proper closing of the chain but it enhances the effectiveness of the sampling procedure by anticipating possible overlaps. This bound is determined by the closest feasible approach between sites 1 and $k$. Bound $\theta_{\text {min }}$ is zero if site 0 cannot "touch" site $k$; otherwise, it is given by

$$
\cos \theta_{\min }=\frac{1}{2 b_{0,1} d_{0, k}}\left(b_{0,1}^{2}+d_{0, k}^{2}-c_{1, k}^{2}\right) .
$$

The value of the upper bound, $\theta_{\max }$, can be determined 
by hypothetically extending all the sites to be regrown in such a way that site 1 be located as far as possible from site $k$. This hypothetical maximal extension is also illustrated in Fig. 4. If site 1 can be "pulled" away in such a way that it can, at least, get totally around site 0 and be placed at the opposite side of site $k$, then $\theta_{\max }=\pi$. Otherwise, we have to identify a site $i^{*}$ which corresponds to the first site of the ungrown section of the chain that touches site 0 once this maximum extension has occurred (site 3 in the example of Fig. 4). Site $i^{*}$ is not necessarily the first site that can touch site 0 ; it is actually the last site, going from site $k-1$ to site 1 , that satisfies

$$
d_{0, i *+1} \geqslant c_{0, i *+1} \text {. }
$$

Note that $d_{0, i^{*}+1}$ refers to the configuration for the hypothetical extension. It can be shown that $d_{0, i^{*}+1}$ is given by

$$
d_{0, i *+1}^{2}=b_{i *, i *+1}^{2}+c_{0, i *}^{2}-\frac{b_{i *, i *+1}}{b_{i *, k}}\left(b_{i *, k}^{2}+c_{0, i *}^{2}-d_{0, k}^{2}\right) \text {. }
$$

Once site $i^{*}$ has been identified, we can compute the angle $\alpha_{i} *, k$, formed by the vectors $\mathbf{c}_{0, i} *$ and $\mathbf{d}_{0, k}$ as

$$
\cos \alpha_{i *, k}=\frac{1}{2 d_{0, k} c_{0, i *}}\left(c_{0, i *}^{2}+d_{0, k}^{2}-b_{i *, k}^{2}\right) .
$$

The same process used to identify $i^{*}$ starting from $k$ has to be followed again to identify the next site tangent to 0 , this time starting from site $i^{*}$. As exemplified in Fig. 4, site $i^{*}-1$ need not be the next tangent site. The value of $\theta_{\max }$ will be given by the sum of angles $\alpha$ produced in this fashion until site 1 is reached. Of course, if at some point this summation is greater than $\pi$ we set $\theta_{\max }=\pi$. Note that, in principle, we need not compute the true $\theta_{\max }$; it suffices to use some value in the range $\left[\theta_{\min }, \theta_{\max }\right]$ to ensure the closing of the chain (although the largest value may increase the overall efficiency of the simulation).

If, for example, we assume that after site $i^{*}$ all sites are tangent to sphere 0 (which is always true for equal-sized sites), we have

$$
\theta_{\max } \leqslant \alpha_{k, i *}+\sum_{j=2}^{i^{*}} \alpha_{j, j-1},
$$

where

$$
\begin{aligned}
\cos \alpha_{j, j-1}= & \frac{1}{2 c_{0, j-1} c_{0, j}}\left(c_{0, j-1}^{2}+c_{0, j}^{2}-b_{j, j-1}^{2}\right), \\
& j=2,3, \ldots, i^{*} .
\end{aligned}
$$

Once the bounds of $\theta$ have been identified, we proceed to sample several orientations of the bond vector $\mathbf{b}_{0,1}$ following a typical CCB prescription. There are two ways of performing such a sampling: (i) mode 1, sample the vector $\mathbf{b}_{0,1}$ uniformly over the whole surface of the sphere around site 0 , rejecting those orientations that fall outside the bounds; (ii) mode 2, sample the vector $\mathbf{b}_{0,1}$ only on the spherical sector bounded by $\theta_{\min }$ and $\theta_{\max }$.

Both of these two approaches require a temporary change in the degrees of freedom used to produce the new

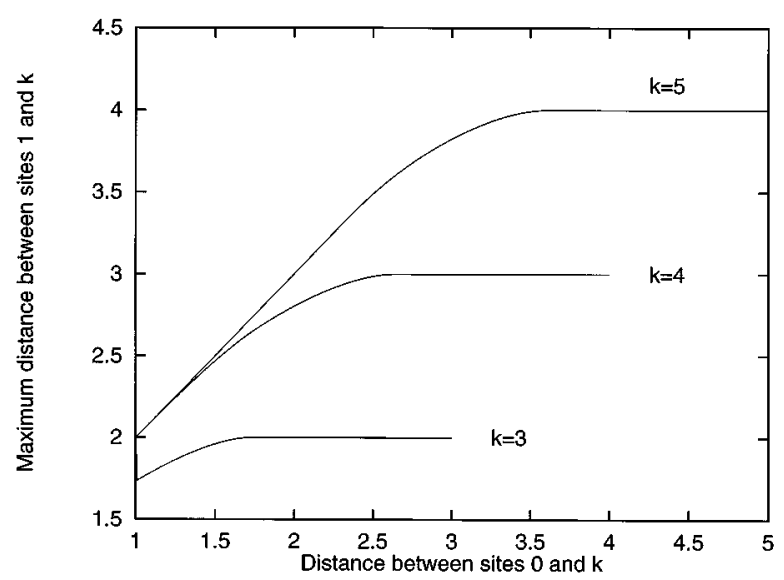

FIG. 5. Maximum-distance functions for freely joined hard-sphere chains. Cases when 2, 3, and 4 intermediate sites are connecting open sites 0 and $k$. Distances in reduced units with respect to the sphere diameter.

configuration; the corresponding Jacobian determinants for this transformation must be incorporated into the acceptance criteria for the move. In Appendix B, we derive the necessary Jacobian determinants to be used along with both sampling schemes.

It turns out that mode-1 sampling can be implemented more easily than mode 2 because the calculations associated with setting up the temporary coordinate system and the evaluation of the bounds of $\theta$ can be avoided altogether if the following alternative procedure is followed. A trial vector $\mathbf{b}_{0,1}$ will possess a valid direction whenever the new calculated distance $d_{1, k}$ satisfies,

$$
d_{\min } \leqslant d_{1, k} \leqslant d_{\max }=d_{\max }\left(k, d_{0, k}\right)
$$

where $d_{\min }=\left(r_{1}+r_{k}\right)$ and $d_{\max }$ are critical distances. In general, $d_{\max }$ is a complicated function of $d_{0, k}$, the number of sites cut, and the geometric characteristics of the chain. The functions required to determine $d_{\max }$ need to be computed only once (at the beginning of the code or in a separate program). In Fig. 5, a few curves for $d_{\max }$ are presented for chains composed of equal-size, tangent hard spheres. Notice that no great accuracy in the value of $d_{\max }$ is required since a smaller value will always be safe. It is important to be aware that by simply setting $d_{\max }=b_{1, k}$ the proper closing of the chain is not always assured.

Mode-2 sampling should not be dismissed entirely, however, because a good acceptance rate will generally require less samples of the growing bond vector than mode- 1 sampling. This might be an important advantage if the calculation of interaction energies is computationally intensive.

Note that the aforementioned procedure is applied to all but the last site to be inserted. In this case $\theta_{\min }=\theta_{\max }$, and the sampling is reduced to producing random variates of the $\phi$ angle. Note also that for the standard CCB move, a $\theta_{\text {min }}$ value could also be identified (although its use may not be justified), while $\theta_{\max }=\pi$ always.

The ECCB method can be implemented as follows.

(i) Select a random chain to be moved. 
(ii) Choose randomly two sites within the chain such that the number of sites in between (including the two chosen sites), $N_{\text {cut }}$, is less than or equal to some specified maximum number $N_{\max } . N_{\text {cut }}$ is the number of sites to be cut and regrown for a particular move.

(iii) If one of the sites in $N_{\text {cut }}$ happens to be an end site, a standard CCB move is performed. If $N_{\text {cut }}=1$ and it is an inner site, the ECCB reduces to a simple crank-shaft move.

(iv) Whether a full ECCB, a CCB or a crank-shaft move is invoked, a site-by-site growth process is carried out using multiple samples for the new bond orientations (as explained in Sec. II A). The total move is accepted with a probability given by Eq. (4), where the appropriate weights are computed as usual from Eqs. (1) and (3). The ratio of the Jacobian determinants is equal to one for the $\mathrm{CCB}$ and crankshaft moves. For the ECCB move this ratio is given in Appendix B.

A random selection of $N_{\text {cut }}$ in the range $\left[1, N_{\max }\right]$ may not be always the best approach. The relative frequency of 1-site, 2-site,.., and $N_{\text {max }}$-site moves can be preset at the beginning of the simulation, thereby providing greater flexibility.

\section{ECCB for branched chains and crosslinked structures}

When a chain has branches, the rearrangement of the branch-linking site presents new difficulties to conventional simulation methods. As mentioned earlier, crank-shaft alone cannot move this site. In principle, a CCB-type of approach could be used if we were to delete and regrow large sections of the chain, including all branches connected to the deleted sites of the main chain. Such a drastic configurational change is unlikely to be successful and would only be useful for short branches and at relatively low densities.

Two methods can be used to propose trial displacements of a branch-linking site.

(i) A "flip" move. The branch-linking site is connected to three sites (two on the main chain and one on the branch) whose centers form a well defined plane. Unless the center of the linking site lies on the same plane, it will be possible to "flip" it over to the other side of the plane (imagine a rotation of the linking site around any of the center-to-center axis formed by two neighboring sites until it "reconnects" the third neighbor site at the other side).

(ii) A combined move. Starting from the branch-linking site, rearrange the entire branch. The linking site is repositioned by a crank-shaft move and subsequent sites are consecutively appended using CCB moves.

A serious disadvantage of the flip move is that only one alternative position is available for the linking site at any one trial. This might limit severely the acceptance rate of this move in a dense phase. A problem with the combined approach is that, to move only one linking site, the whole branch has to be moved. This method will, consequently, have many of the shortcomings of the conventional CCB whenever the branches are long or the density is high.

A much better method can be devised by specializing the ECCB to perform moves of a branch-linking site. In principle, there are several ways of implementing such a move. We will limit our discussion to only two of them; this should
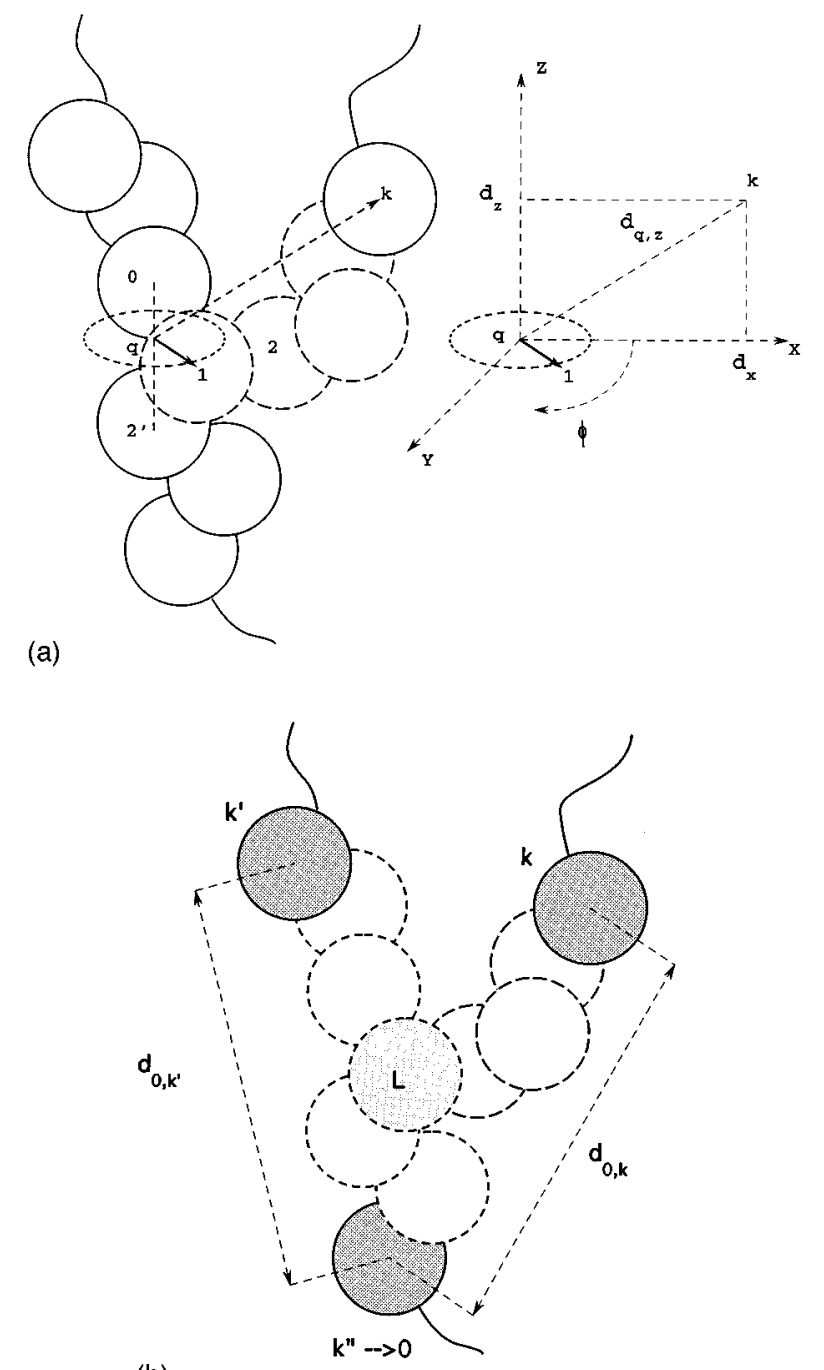

(b)

FIG. 6. (a) Local coordinate system for the rearrangement of a branchlinking site using ECCB moves; (b) rearrangement of arbitrary chain sections around a branch-linking site.

suffice to illustrate the general approach. The nomenclature for the first application is illustrated in Fig. 6(a). Site 1 is the branch-linking site. Sites from 2 up to $k-1$ are also to be relocated, and belong to either the main chain or the branch. Sites 0 and $2^{\prime}$ are to be kept fixed; they constitute, along with site 2 , the three neighbors of site 1 . The sites cut are to be regrown in sequence, from 1 to $k-1$. To regrow site 1 we need to define some bounds to the rotation angle that will ensure that site $k-1$ will ultimately be able to connect with site $k$.

We choose the $z$ axis to lie along the normal vector to the rotation plane of site 1 (centered at point $q$ ). The $x$ axis is chosen to lie along the perpendicular projection of vector $\mathbf{d}_{q, j}$ on the $z$ axis. With this particular choice, $\phi=0$ defines the closest approach of sites 1 and $k$, and $\phi= \pm \pi$ defines their maximum separation, $d_{\text {sup }}$, which is given by

$$
d_{\text {sup }}=\left[d_{z}^{2}+\left(d_{x}+d_{q, 1}\right)^{2}\right]^{1 / 2},
$$


where $d_{z}$ and $d_{x}$ denote the projections of vector $\mathbf{d}_{q, k}$ on the $z$ and $x$ axis, respectively. If $d_{\max }$ $=\min \left[d_{\text {sup }} ; d_{\max }\left(k, d_{0, k}\right) ; d_{\max }\left(k, d_{2, k}\right)\right] \leqslant b_{1, k}$, then, $\left|\phi_{\max }\right|$ $=\pi$, otherwise we have

$$
\cos \phi_{\max }=\frac{1}{2 d_{x} d_{q, 1}}\left(d_{q, k}^{2}+d_{q, 1}^{2}-b_{1, k}^{2}\right) \text {. }
$$

A lower bound (in absolute value) can also be identified in this move, which arises whenever $c_{1, k}>d_{q, k}$, and is given by

$$
\cos \phi_{\min }=\frac{1}{2 d_{x} d_{q, 1}}\left(d_{q, k}^{2}+d_{q, 1}^{2}-c_{1, k}^{2}\right) .
$$

The new position of site 1 is found by choosing a random value of $\phi$ such that $\phi_{\min } \leqslant|\phi| \leqslant \phi_{\max }$.

An even simpler prescription to move site 1, in the spirit of mode-1 sampling of the ECCB move, consists of proposing random rotations of $\phi$ on $[-\pi,+\pi]$ and then, if $c_{1, k}>d_{1, k}>d_{\text {max }}$, rejecting the trial move; otherwise the move is considered valid.

In any case, once site 1 has been repositioned (by the usual CCB-type multisampling), we can proceed to regrow the rest of the cut sites using our ECCB prescription. Note also that this move becomes the "flip" move described earlier if only site 1 is cut $\left( \pm \phi=\phi_{\min }=\phi_{\max }\right)$, while it becomes the "combined" method if site $k-1$ is an end site.

This method can be easily extended for an arbitrary deletion of sites around the linking site (labeled $L$ ) as illustrated in Fig. 6(b). If the chain is being left "open" at three locations, say sites $k, k^{\prime}$, and $k^{\prime \prime}$, the regrowing process could proceed in two steps as follows.

(i) Regrow first one "thread" of the chain until reaching the linking-site, for instance, from site $k$ " to site $L$. This is accomplished with a variation of the ECCB move, where the trial position of a growing-site $j$ is now considered valid if both $d_{j, k}$ and $d_{j, k^{\prime}}$ are within their corresponding bounds [see Eq. (11)].

(ii) Once the linking site has been regrown, we can proceed to close the open sites $\left(k\right.$ and $\left.k^{\prime}\right)$ in any order, using ECCB moves.

If site $L$ is the last to be regrown, it can take one of the two positions corresponding to $\phi= \pm \phi_{\min }= \pm \phi_{\max }$ as computed from Eq. (13) or (14) with $L \leftrightarrow 1$. Note that the proper Boltzmann weights calculated according to Eq. (1), have to be determined for each regrown site, regardless of the stage of the regrowing process. The Jacobian determinant for this type of move is also given in Appendix B.

The method described above is equally applicable to branched chains and crosslinked structures with three-branch linking sites. Extension of the method to $n$-branch linking sites with $n \geqslant 4$ is straightforward.

\section{E. The HOLE method}

Conformational changes in the segments of a chain are more likely to occur whenever large void spaces are found. Since at high densities such spaces occur only rarely, conventional Monte Carlo methods are unable to produce significant conformational rearrangements. One way of fa-

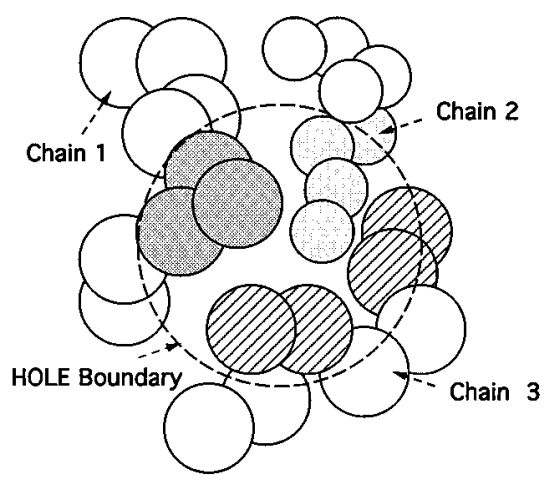

(a)

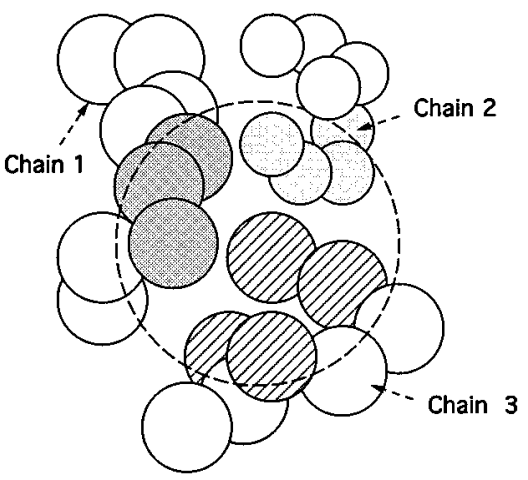

(b)

FIG. 7. Illustration of the operation of the HOLE method. (a) Local configuration before the move; (b) configuration after the move.

voring these internal changes is to create artificial, temporary holes in the system by deleting sites located within a specified spherical region. It might be advantageous to implement a preferential sampling technique ${ }^{23}$ to rearrange just some of the sites within the hole. By confining the sites cut to just one region, neighboring sites have a tendency to "exchange" locations. In a closely packed structure, this exchange may result in more drastic changes than single moves.

With the HOLE method, sites located within the hole can be end segments or inner segments from different chains. In fact, the same chain may be cut at several distinct locations. The principle of operation of the method is illustrated in Fig. 7.

To ensure microscopic reversibility, no regrown segments are allowed to leave the boundaries (the old and new configurations must be accessible to each other through analogous sampling and regrowing processes). This additional constraint tends to decrease the efficiency of the moves. A simple way of increasing the chances of producing successful rearrangements is by limiting the maximum number of sites to be present in the hole. In this way, rearrangements are attempted only when the sites in the hole have a "favorable" disposition.

The ECCB move can be easily implemented to perform the regrowing process. A typical scheme could operate as follows.

(i) Select a (random) point inside the simulation box.

(ii) Remove a number of sites (chosen at random) within a radius of $R_{\text {cut }}$ of that point. 
(iii) Identify the sites cut by "set" numbers. Sites belonging to a given set must be contiguous sites on the same chain. One chain can have more than one set of sites in the hole.

(iv) Regrow sites in a random sequence using ECCB moves. Any trial position that places the center of a site outside the hole boundaries must be rejected. For each set $j$ regrown, compute and store its corresponding weight according to

$$
W_{\text {set }_{j}}=\prod_{i=1}^{N_{\text {sites }_{j}}} w_{i},
$$

where $w_{i}$ is computed from Eq. (1) and $N_{\text {sites }_{j}}$ is the number of sites in the $j$ th set of sites. Furthermore, each set will have associated to it a Jacobian determinant which must be evaluated as indicated in Appendix B.

(v) In case that a complete molecule was deleted in the hole (i.e., a small solvent molecule), a number of trial displacements for one of the end sites is attempted (with magnitude and direction chosen at random). Disregarding trial positions outside the hole, one trial position is chosen with probability given by Eq. (1) and its weight is included into the acceptance criteria as usual. The following sites (if existing) are then regrown using standard CCB moves.

(vi) Repeat the regrowing process but this time to recover the old, original configuration. This is done to compute the analogous weights required in Eq. (15) for the old configuration.

(vii) The move is accepted with probability

$$
P_{\text {HOLE }}=\min \left(1, \frac{J^{\text {new }}}{J^{\text {old }}} \frac{W^{\text {old }}}{W^{\text {new }}} \frac{P_{\text {new }}}{P_{\text {old }}}\right),
$$

where

$$
\begin{gathered}
J=\prod_{j=1}^{N_{\text {sets }}} J_{\text {set }_{j}}, \\
W=\prod_{j=1}^{N_{\text {sets }}} W_{\text {set }_{j}} .
\end{gathered}
$$

As shown later, a naive implementation of the HOLE method can be inefficient. The method should be modified to take advantage of the particular characteristics of a given system. We illustrate this point by simulating a system of polymer chains dissolved in its monomer. The HOLE method can be used for chain-related moves only, one chainsite at a time, as follows.

(i) Choose the segment $i$ to be rearranged. Locate the center of the HOLE at (i) the center of the previous site if $i$ is an end site, or (ii) at the center of the crank-shaft rotation plane of site $i$ if it is an inner site.

(ii) Make the HOLE radius large enough to include totally or partially site $i$ (at least its center must be included). Identify only solvent molecules within the HOLE and chose some of them at random for subsequent rearrangement. (iii) Proceed with the method as usual by regrowing first the chain site and then the solvent molecules (using CCB sampling).

In this implementation, we locate the hole in a favorable position for a one-chain-site rearrangement and we take advantage of the greater "mobility" of solvent molecules over chain segments.

\section{TEST OF THE METHODS}

The methods proposed in this work satisfy microscopic reversibility by construction. However, in order to verify that our sampling methods (and the corresponding Jacobian expressions) and codes are correct, we have performed extensive numerical tests, some of which are briefly discussed in this section.

We tested the ECCB method by performing Monte Carlo simulations of a 16-mer "phantom" chain and an 8-mer tangent-hard-sphere chain in vacuum. The sites of the phantom chain consist of simple points (i.e., excluded volume effects are ignored). The 8-mer consists of equal-size hard spheres. In both cases, sites are connected by rigid bonds of equal length.

For the phantom chain, the probability density distributions of the bond-bond and dihedral angles ${ }^{8}$ are independent of the site position. It can be easily shown that these probability densities are given by

$$
\begin{aligned}
& \rho\left(\angle_{\text {bond-bond }}\right)=\frac{1}{2} \sin \left(\angle_{\text {bond-bond }}\right), \\
& \rho\left(\angle_{\text {dihedral }}\right)=\frac{1}{\pi} .
\end{aligned}
$$

Both angles have been defined in the range $[0, \pi]$ only. Figure 8 shows the results of a simulation of $3 \times 10^{6}$ ECCB trial moves with mode- 1 sampling. The results obtained by ignoring the Jacobian correction are also plotted for comparison. To avoid the masking effect that one-site moves could introduce in the results (since their Jacobian correction is one) only three-site moves were used for these simulations. Clearly, the Jacobian correction is needed to ensure a completely unbiased sampling of the configurational space and thus guarantee microscopic reversibility. Similar results are obtained using mode- 2 sampling.

Figure 9 shows analogous results (three-site moves only) using mode-2 sampling for the hard-sphere 8-mer chain in vacuum. These simulations include excluded volume effects. Analytical solutions for the angle probability densities are not available. The reference curves have been obtained by the standard CCB method. These figures illustrate more dramatically the important consequences of the Jacobian correction in ECCB moves.

A number of additional tests have been performed to ensure the validity of our ECCB moves and codes. Limitations of space, however, preclude their discussion in this manuscript. Note that, in particular, it has been thoroughly tested that the last sites to be appended do not tend to 'line up' in order to close the chain. A near line-up configuration corresponds to the case of having $\theta_{\max } \approx \theta_{\min }$ for the growing sites involved, i.e., the valid sampling space is very small (see Fig. 3) and, thus, the chances of accepting the move are 


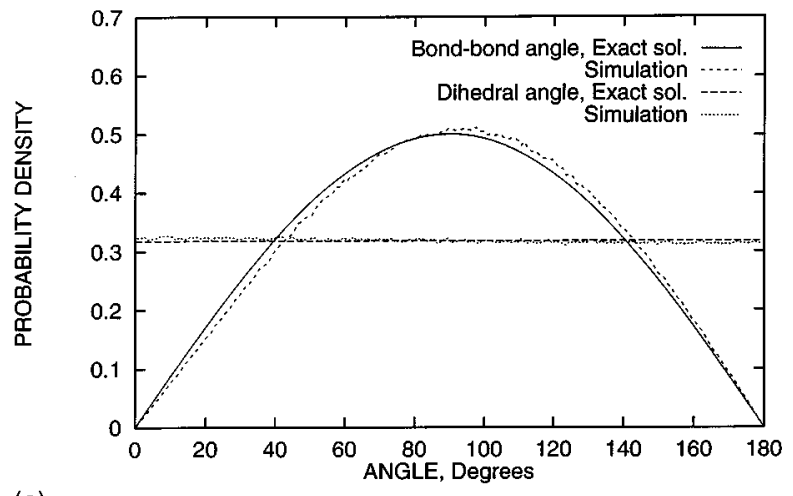

(a)

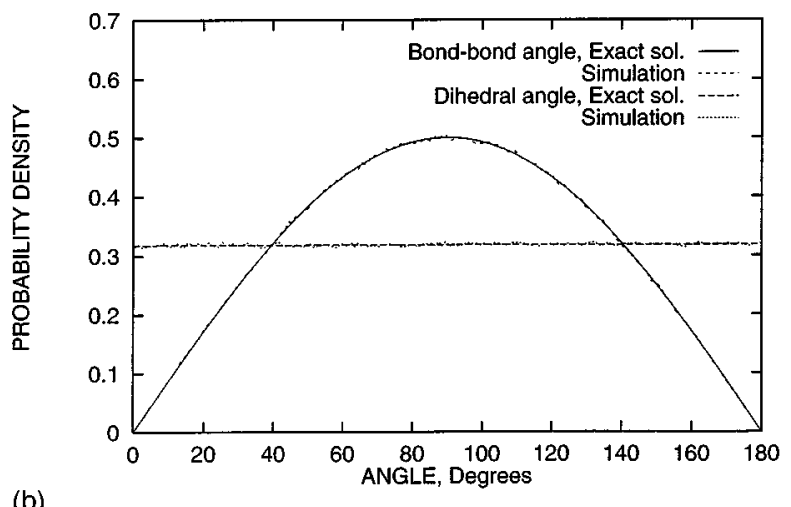

(b)

FIG. 8. Bond-bond and dihedral angle distributions from the analytical solution and MC ECCB-3 (mode-1 sampling) simulations for a phantom chain of 10 segments. (a) Without Jacobian of transformation in acceptance criteria, (b) with Jacobian of transformation in acceptance criteria.

negligible. In fact, a factor proportional to the fraction of valid sampling space is associated to the probability of accepting any proposed configuration, and appears explicitly in the Jacobian of mode-2 sampling [Eq. (B8)] and implicitly in the Rosenbluth weights of mode-1 sampling (in which case the chain closing attempt may fail).

To test the HOLE method we performed NVT simulations of a system consisting of 32 hard-sphere chains of 16 tangent-equal-size sites. A low packing fraction was used $(\eta=0.1)$ to facilitate successful moves. The reference curves for the angular density distribution functions at these conditions were generated by conventional simulation techniques. We performed an analogous simulation using the HOLE method, where the radius of the spherical hole was set to one fourth of the box length. The average number of spheres within the hole was approximately 10. The results for the bond-bond angle are given in Fig. 10(a). For completeness, we also show the results obtained when moves outside the hole are also allowed; these calculations confirm that moves that place the sites outside the hole must be avoided.

A test of the HOLE method as implemented for the case of a dense, concentrated polymer solution $(\eta=0.35)$ is illustrated in Fig. 10(b). The system consisted of twenty freely jointed hard sphere 16-mers dissolved in 640 hard-sphere solvent molecules having a diameter equal to that of the chain sites.

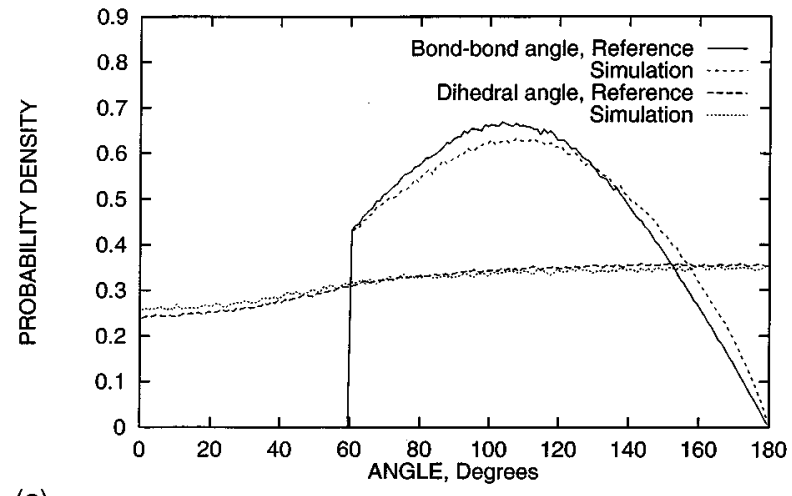

(a)

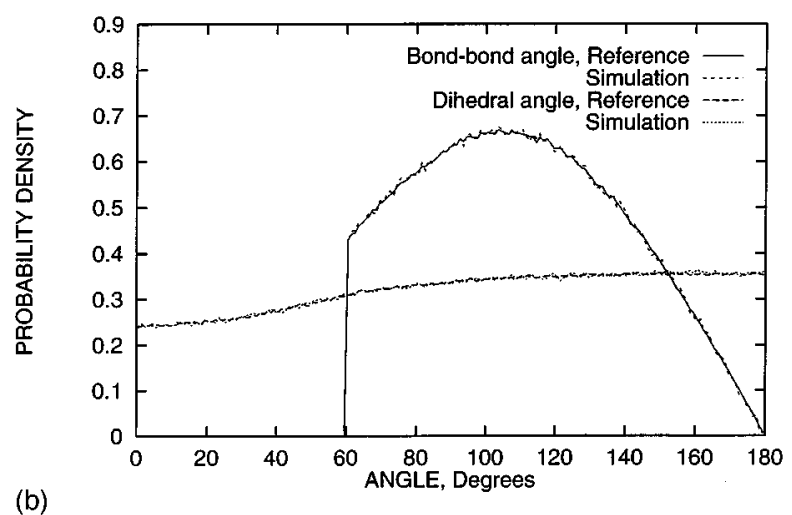

FIG. 9. Bond-bond and dihedral angle distributions from the $\mathrm{CCB}$ and ECCB-3 (mode-2 sampling) simulations for an 8-mer hard-sphere chain. (a) Without Jacobian of transformation in acceptance criteria; (b) with Jacobian of transformation in acceptance criteria.

\section{COMPUTATIONAL DETAILS}

The simulations were performed in cubic simulation boxes with the usual periodic boundary conditions. For simplicity, in all cases our system consisted of hard-sphere chains with tangent-equal-size beads.

\section{A. Simulation performance}

To assess the performance of the methods proposed here, we run simulations in the NVT ensemble and we measure the following time correlation functions. ${ }^{9}$

The bond orientation autocorrelation function. It provides information on how fast local rearrangements are taking place as the simulation proceeds. It is given by

$$
b c f(t)=\left\langle\mathbf{u}_{i}(t) \cdot \mathbf{u}_{i}(t+\delta t)\right\rangle_{i, k},
$$

where the averaging is over all $i$ bonds in the system and over $k$ Monte Carlo steps, and where $\mathbf{u}$ denotes the unit bond vector.

The end-to-end orientation autocorrelation function. It provides information about long-time rearrangements of the chain molecules. It is given by

$$
e c f(t)=\left\langle\mathbf{e}_{j}(t) \cdot \mathbf{e}_{j}(t+\delta t)\right\rangle_{j, k},
$$

where the averaging is over all $j$ chains in the system and over $k$ Monte Carlo steps, and where $\mathbf{e}_{j}$ denotes the unit vector along the line that joints the first and last sites of the $j$ th chain. 


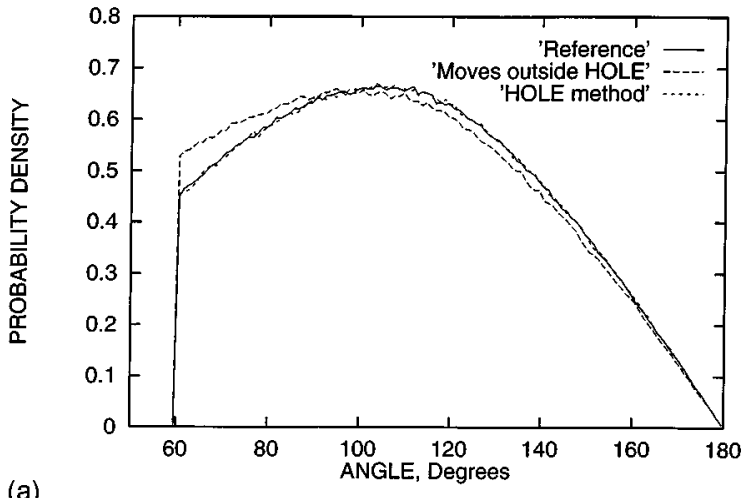

(a)

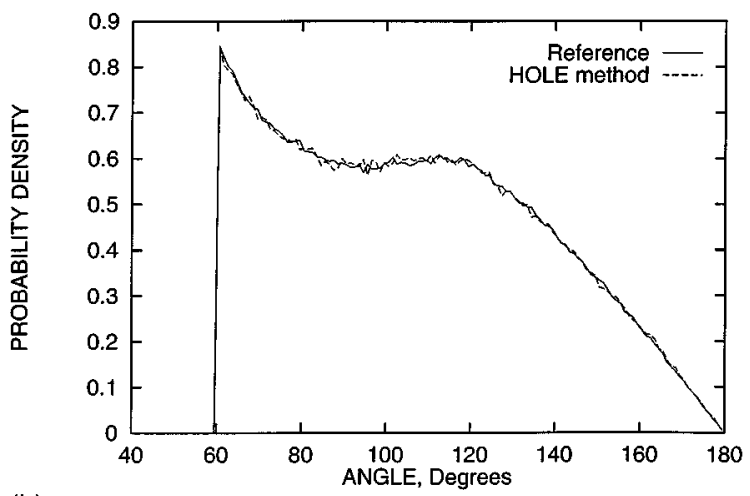

(b)

FIG. 10. Bond-bond angle distribution functions from crank-shaft and HOLE simulations for hard-core systems. (a) 16-mer chains at a packing fraction of $\eta=0.1$, showing the effect of allowing rearrangements outside the hole boundaries; (b) mixture of $33 \%$ volume $16-\mathrm{mer} /$ monomer at $\eta=0.35$.

For these simulations the system consisted of 32 chains of 16 beads; two densities $(\eta=0.30$ and $\eta=0.40)$ were studied. The chain length of 16 was selected to yield moderately fast relaxation times for the end-to-end vector orientation. A packing fraction of $\eta \approx 0.3$ corresponds to typical conditions encountered in polymer melts.

A polymer-solvent system was also investigated to compare the performance of the HOLE method (as suggested in Section II E) to conventional crank-shaft moves for innerchain segments. The system consisted of 16-mer hard chains dissolved in monomer for a $33 \%$ volume concentration and $\eta=0.35$. For the chain-segment moves, at most one solvent molecule was chosen to be rearranged from those located within the hole. The hole radius was set equal to the site diameter for inner chain-site moves; it was $10 \%$ bigger for end-site moves.

There are many ways to increase the time-efficiency of Monte Carlo simulations, such as the implementation of neighbor lists, linked-cell structures, etc. ${ }^{23}$ For our work we use a mini-cell structure in which the cell size is small enough to accommodate at most one site. It is important to point out that the time-efficiency of an algorithm is not only machine dependent but it also depends on programming style, implementation of special techniques, and characteristics of the system. For this reason, the evolution of our simulations is generally reported in Monte Carlo steps; we restrict the use of real CPU time to a few illustrative calculations (the reported values correspond to a Silicon Graphics XS24 4000 workstation).

\section{B. Isobaric-isothermal ensemble simulations}

The performance of the new methods for simulation of long chains at high densities is tested by calculating the compressibility factor as a function of packing fraction for two homopolymer and two binary hard-core systems. The homopolymer systems have chain lengths of 16 and 51 segments respectively. The binary systems consist of an 8-mer/ monomer blend at $88.9 \%$ chain concentration (volume) and a 16-mer/monomer blend at $50 \%$ concentration, respectively. Note that no Monte Carlo PV data exist for homopolymer hard-sphere chains of more than 16 sites $^{14,24}$ and for hardcore monomer-polymer mixtures with chains of more than eight sites. ${ }^{13}$ The 16-mer homopolymer system and the 8-mer/monomer system are included to provide additional PV data in the high-density range only, where previous simulation data have been inconsistent or missing.

The values of the reduced pressure ${ }^{23} P^{*}$ were specified and the values of $\eta$ were obtained from NPT simulations. For our systems, the compressibility factor is given by

$$
Z=\frac{\pi}{6} \frac{N_{s}}{N_{c}} \frac{P^{*}}{\eta},
$$

where $N_{s}$ is the total number of sites and $N_{c}$ is the number of chains.

For the homopolymer simulations, ECCB moves were performed with a maximum of four sites cut per trial $\left(N_{\text {samp }}\right.$ was adjusted according to the cut size to yield an overall acceptance rate of 10-20\%). For mixture simulations, we implemented the HOLE method described in Sec. II E ( $N_{\text {samp }}$ was adjusted to get $\sim 20 \%$ acceptance rate for chain-site moves). Random volume changes were attempted approximately every $N_{s} / 2$ molecular moves and accepted following suitable criteria. ${ }^{23}$ The maximum volume change was adjusted to get a 40-50\% acceptance rate. The values of compressibility factor reported in this work correspond to the average of two independent simulations (started from different initial configurations). At high densities and for long chains, each of these simulations (started from a lower density) required one billion moves just to achieve equilibration.

For concreteness, in all simulations that involved the use of ECCB moves, only the results of mode- 1 sampling are reported; results using mode-2 sampling are comparable.

\section{RESULTS AND DISCUSSION}

Figure 11 shows the bond and end-to-end relaxation functions for the homopolymer system at $\eta=0.3$ for different moves. ECCB-3 denotes that only three-site moves were used for the simulation. For the standard CCB, up to eightsite rearrangements were allowed. For both the $\mathrm{CCB}$ and ECCB-3 moves, we used a value of $N_{\text {samp }}=6$ [see Eq. (1)]. A value of $N_{\text {samp }}=12$ was used for a HOLE diameter of 1.2 units (site diameter $=1$ ) and no maximum number of regrowing sites was preset. The average number of sites effectively regrown was 2.9 for the HOLE method and less than 1.7 for 


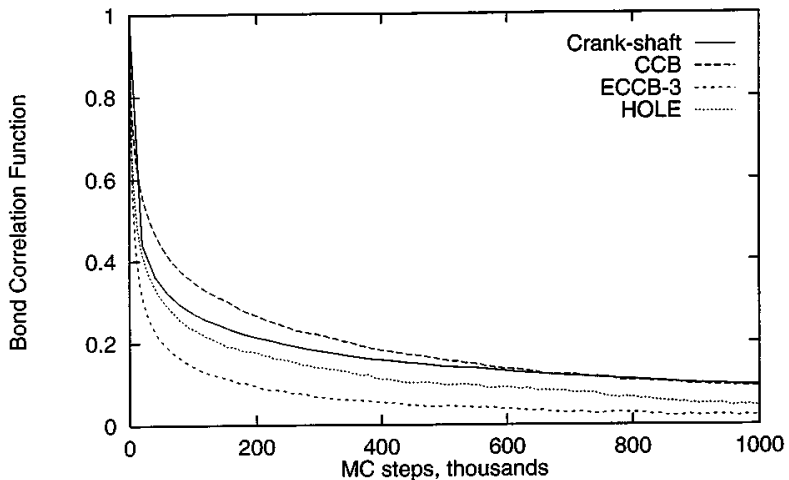

(a)

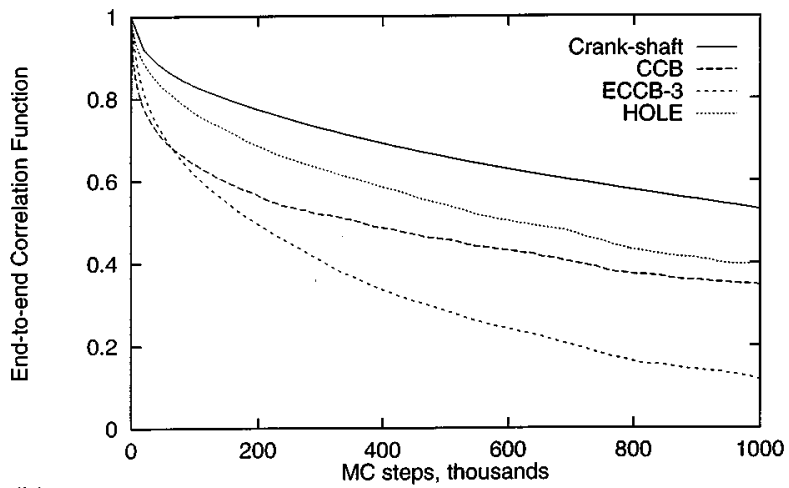

(b)

FIG. 11. Correlation functions for crank-shaft, CCB, ECCB-3, and HOLE moves for a system of 16-mer hard-sphere chains at $\eta=0.3$. (a) Bond autocorrelation function; (b) end-to-end autocorrelation function.

CCB moves. The average acceptance rates were $29 \%$ for crank-shaft, $5.8 \%$ for ECCB-3, 8.2\% for CCB moves, and $11.2 \%$ for the HOLE method.

ECCB-3 produces the fastest decay of both correlation functions. Since the chains are relatively short, standard CCB moves are somewhat effective, specially for decorrelating the end-to-end orientations (as expected). However, after a fast initial decay, the end-to-end correlation curve for the CCB moves slows down significantly, presumably as a result of the limited rearrangement of innermost sites. These curves suggest that, for pure homopolymers, the HOLE method is less effective than pure ECCB moves.

Figure 12 shows the corresponding curves for two-site, three-site and four-site ECCB moves. For all ECCB moves a value of $N_{\text {samp }}=6$ was used, which may not be optimal for all of them. These results show that the ECCB move has a rather consistent performance for different cut lengths.

Figure 13 illustrates the effect of incorporating a configurational-bias into the crank-shaft moves. $N_{\text {samp }}=1$ corresponds to a simple crank-shaft move. It is observed that at these conditions a value of $N_{\text {samp }}=3$ improves the performance of crank-shaft moves, although the effect is small at the local level. Configurational bias sampling will have a more pronounced positive effect at higher densities.

Some selected curves of time correlation for different moves and using a real time scale are shown in Fig. 14. In spite of the highly variable nature of this comparison, some qualitative remarks are in order. While ECCB and crank-

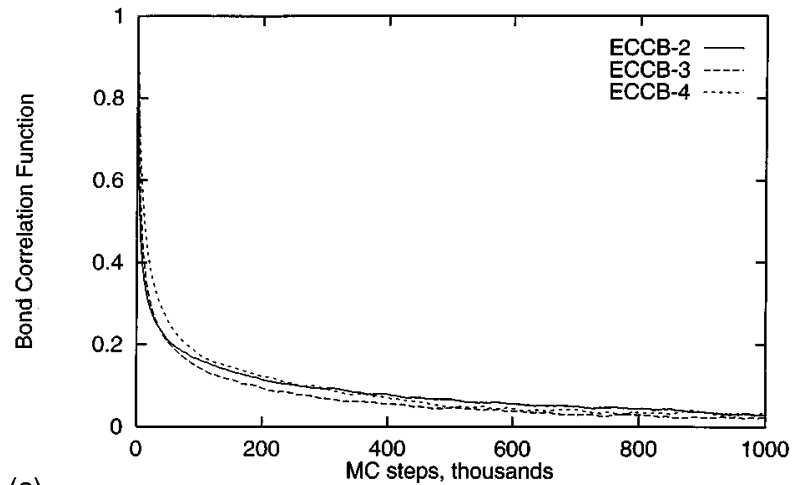

(a)

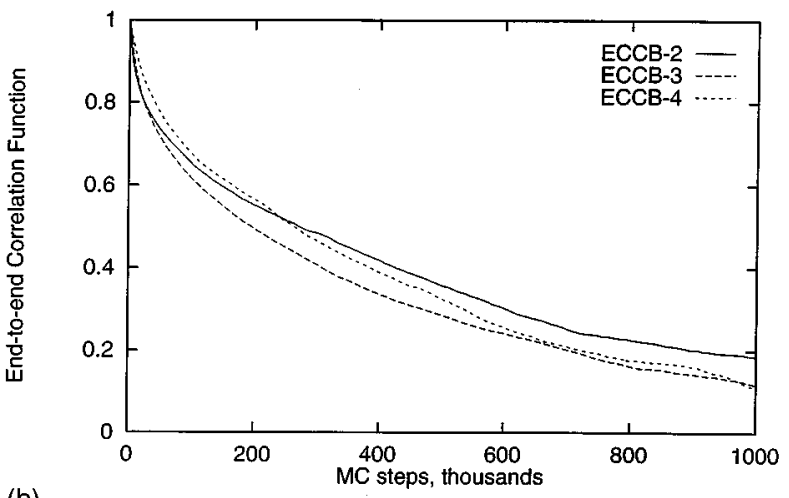

(b)

FIG. 12. Correlation functions for ECCB moves regrowing exclusively 2, 3 , or 4 sites in a system of 16-mer hard-sphere chains at $\eta=0.3$. (a) Bond autocorrelation function; (b) end-to-end autocorrelation function.

shaft moves compare closely at the local level of rearrangement (bond orientation correlations), the former yield a significantly faster relaxation of the longer scale end-to-end orientation vector. These results also suggest that the HOLE method would not offer an advantage over the other methods for simulation of homopolymer melts. Results of additional simulations at a packing fraction of $\eta=0.4$ exhibit similar qualitative features.

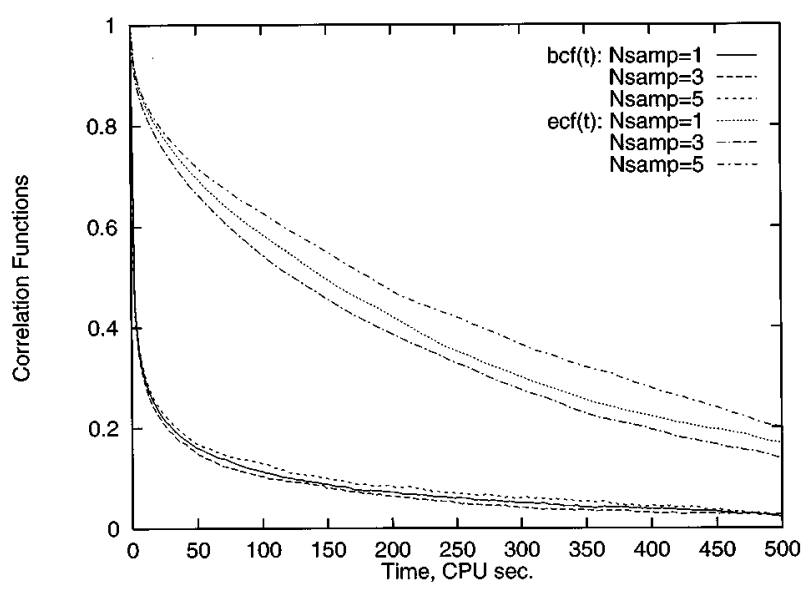

FIG. 13. Bond and end-to-end autocorrelation functions for crank-shaft moves for $N_{\text {samp }}=1,3$, and 5 . 

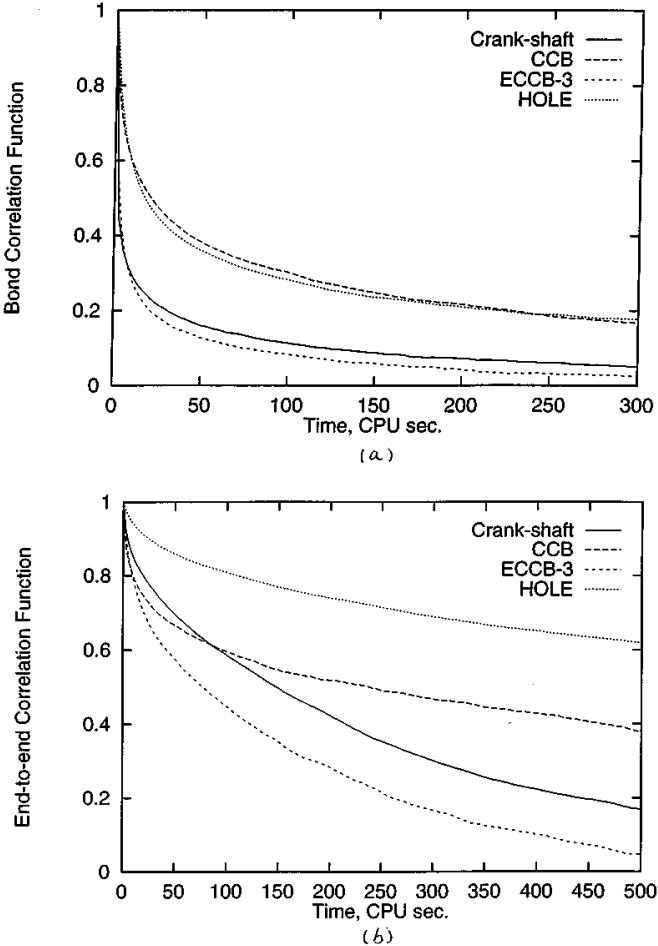

FIG. 14. Correlation functions for crank-shaft, CCB, ECCB-3, and HOLE moves on a real time scale for a system of 16-mer hard-sphere chains at $\eta=0.3$. (a) Bond autocorrelation function; (b) end-to-end autocorrelation function.

The time correlation results for the binary system are shown in Fig. 15. For chain-segment moves, a value of $N_{\text {samp }}=5$ was used when regrowing a chain site and a value of $N_{\text {samp }}=25$ was used to reposition the solvent molecule (if no solvent molecule was present in the HOLE, $N_{\text {samp }}=1$ was used for the chain site). A large value of $N_{\text {samp }}$ for the solvent is necessary because at high densities most of the hole space is generally occupied (by the regrowing chain site and its neighbors). Note, however, that no attempts have been made

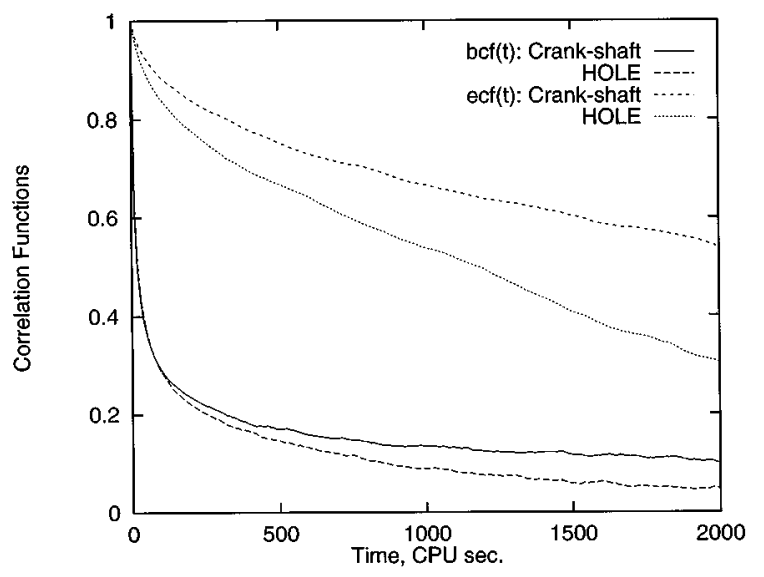

FIG. 15. Correlation functions for crank-shaft and HOLE moves on a real time scale for a hard-core system of 50\% (volume) 16-mer/monomer mixture at $\eta=0.35$.
TABLE I. Simulation results for homopolymer hard-sphere chains. The MC steps correspond to the averaging part of the calculation and apply to each of two independent simulations.

\begin{tabular}{ccrcrc}
\hline \hline$n$-mer & $P^{*}$ & $N_{c}$ & $\eta$ & \multicolumn{1}{c}{$Z$} & MC steps \\
\hline 16 & 2.29 & 80 & $0.368 \pm 0.0025$ & 52.2 & $300 \times 10^{6}$ \\
16 & 3.86 & 100 & $0.417 \pm 0.0017$ & 77.6 & $350 \times 10^{6}$ \\
16 & 6.28 & 100 & $0.463 \pm 0.0015$ & 113.7 & $400 \times 10^{6}$ \\
& & & & & \\
51 & 0.05 & 20 & $0.111 \pm 0.0017$ & 12.0 & $70 \times 10^{6}$ \\
51 & 0.26 & 40 & $0.198 \pm 0.0020$ & 35.1 & $120 \times 10^{6}$ \\
51 & 0.60 & 40 & $0.258 \pm 0.0032$ & 61.6 & $150 \times 10^{6}$ \\
51 & 1.10 & 60 & $0.306 \pm 0.0016$ & 96.0 & $200 \times 10^{6}$ \\
51 & 1.80 & 60 & $0.348 \pm 0.0014$ & 138.3 & $250 \times 10^{6}$ \\
51 & 3.20 & 60 & $0.403 \pm 0.0012$ & 212.0 & $350 \times 10^{6}$ \\
51 & 5.20 & 60 & $0.447 \pm 0.0010$ & 310.6 & $500 \times 10^{6}$ \\
\hline \hline
\end{tabular}

to optimize these initial guesses of $N_{\text {samp }}$. The improvement of HOLE moves over crank-shaft moves is significant at the long-term level. To relax the system to a $60 \%$ of the initial configuration of end-to-end orientation vectors, the HOLE method needs less than half the time required by crank-shaft moves. Furthermore, the difference between the relaxation curves for both methods tends to increase with time.

A number of parameters affect the time-performance of CCB-type moves. The single most important factor is probably the choice of $N_{\text {samp }}$. If it is too large, time is wasted generating many alternative "valid" positions for a growing site when only one of them is used. On the other hand, too few samples may fail to generate enough successful moves. The optimum value of $N_{\text {samp }}$ depends on operating conditions (i.e., density), geometry of the sites, number of growing sites, etc. Additional factors play a role in the efficiency of the HOLE method, such as the order in which the sets are regrown, the maximum preset number of sites within the hole, geometry and size of the hole, etc. The avoidance of moves outside the hole also decreases the effectiveness to the method. A thoughtful choice of the hole geometry, and of internal and external preferential sampling schemes could alleviate this problem. It is beyond the scope of this paper to analyze the effects of all these factors which are highly system dependent.

The real benefit of the HOLE method resides in its applicability to some important systems. The results for the solvent-polymer system illustrate a more specialized application of the HOLE method. It is anticipated that the improvement will be more significant at higher densities and for solvent molecules having a diameter different from that of the chain sites. In these systems, chain rearrangements are severely hindered by small molecules filling the surrounding voids. For this and some other asymmetric systems for which conventional methods are very ineffective, the HOLE method provides an efficient means of producing collective molecular rearrangements. The HOLE method also provides an interesting framework for development of new simulation techniques such as those suggested in our concluding section.

The results of the NPT simulations are summarized in Tables I and II. The compressibility curve for the different systems are given in Figs. 16-19. Overall, our data for 51- 
TABLE II. Simulation results for $n$-mer/monomer mixtures. The MC steps correspond to the averaging part of the calculation and apply to each of two independent simulations.

\begin{tabular}{ccccrc}
\hline \hline & & $N_{c}$ & & & \\
$n$-mer & $P^{*}$ & $(n$-mer/1 $)$ & $\eta$ & \multicolumn{1}{c}{$Z$} & MC steps \\
\hline 8 & 1.10 & $80 / 80$ & $0.2850 \pm 0.0020$ & 9.09 & $300 \times 10^{6}$ \\
8 & 3.60 & $100 / 100$ & $0.4003 \pm 0.0020$ & 21.19 & $350 \times 10^{6}$ \\
8 & 5.90 & $100 / 100$ & $0.4499 \pm 0.0019$ & 30.90 & $400 \times 10^{6}$ \\
16 & 0.17 & $15 / 240$ & $0.0994 \pm 0.0008$ & 1.69 & $100 \times 10^{6}$ \\
16 & 0.61 & $30 / 480$ & $0.1994 \pm 0.0018$ & 3.02 & $150 \times 10^{6}$ \\
16 & 1.03 & $30 / 480$ & $0.2497 \pm 0.0020$ & 4.07 & $300 \times 10^{6}$ \\
16 & 1.70 & $40 / 640$ & $0.300 \pm 0.0030$ & 5.59 & $350 \times 10^{6}$ \\
16 & 2.70 & $40 / 640$ & $0.3495 \pm 0.0020$ & 7.61 & $350 \times 10^{6}$ \\
16 & 4.30 & $40 / 640$ & $0.3957 \pm 0.0019$ & 10.70 & $400 \times 10^{6}$ \\
16 & 6.70 & $40 / 640$ & $0.4370 \pm 0.0014$ & 15.11 & $400 \times 10^{6}$ \\
\hline \hline
\end{tabular}

mers (Fig. 16) and 16-mers (Fig. 17) are consistent with those of Gao and Weiner. ${ }^{24}$ However, some small deviations are observed, particularly at high densities. Our Monte Carlo results and those obtained by others for the 16-mer system, ${ }^{15,21}$ indicate that Gao and Weiner tend to slightly overpredict the compressibility factor at intermediate densities, while the opposite holds true at very high densities. Note also that Gao and Weiner had to resort to an indirect method to arrive at their results. They used molecular dynamics to simulate a freely joined "effective" hard-sphere chain model, where the parameters of a continuous noncovalent potential are selected to approximate the PV behavior of the hard-sphere fluid. Their results are therefore approximate and, unfortunately, their approach cannot be applied in its present form to simulation of copolymers, branched polymers, or blends. For the 16-mer homopolymer system, our

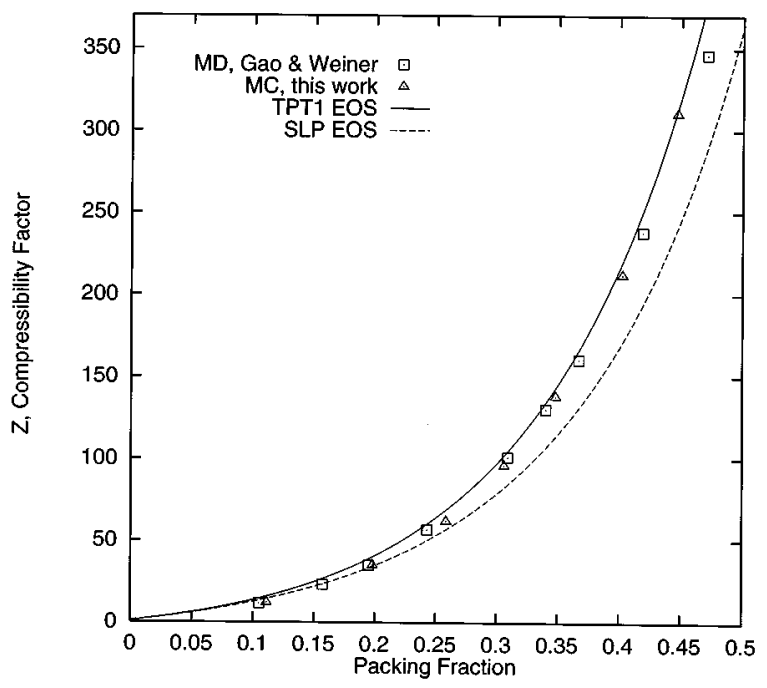

FIG. 16. Compressibility factor vs packing fraction for freely jointed tangent hard-sphere chains of 51 segments. Results of MD simulations of Gao and Weiner (Ref. 24) ( $\square$ ), MC simulations of this work $(\triangle)$, the first-order thermodynamic perturbation theory (TPT1) (solid line) and the SongLambert-Prausnitz (SLP) (dashed line) equations of state.

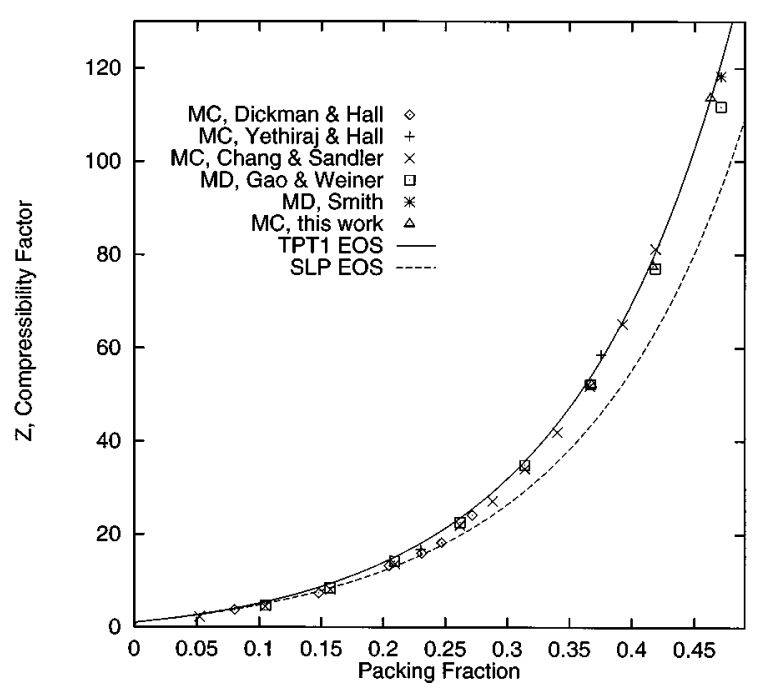

FIG. 17. Compressibility factor vs packing fraction for freely jointed tangent hard-sphere chains of 16 segments. Simulation results of Dickman and Hall (Ref. 15) $(\diamond)$, Yethiraj and Hall (Ref. 14) (+), Chang and Sandler (Ref. 21) (×), Gao and Weiner (Ref. 24) ( $\square$ ), Smith (Ref. 38) (*), and this work $(\triangle)$. Predictions of the first-order thermodynamic perturbation theory (TPT1) (solid line) and the Song-Lambert-Prausnitz (SLP) (dashed line) equations of state.

highest density point is in better agreement with an unpublished result obtained by $\mathrm{Smith}^{38}$ using molecular dynamics.

To illustrate the relevance of the methods and the results presented in this work, we compare our results to the predictions of recently developed equations of state. We have chosen two models that appear to be representative of two different but widely used approaches for development of equations of state. Figures 16-19 show the predictions of the equations of state developed by Chapman et al. ${ }^{16}$ (based on the first-order thermodynamic perturbation theory of

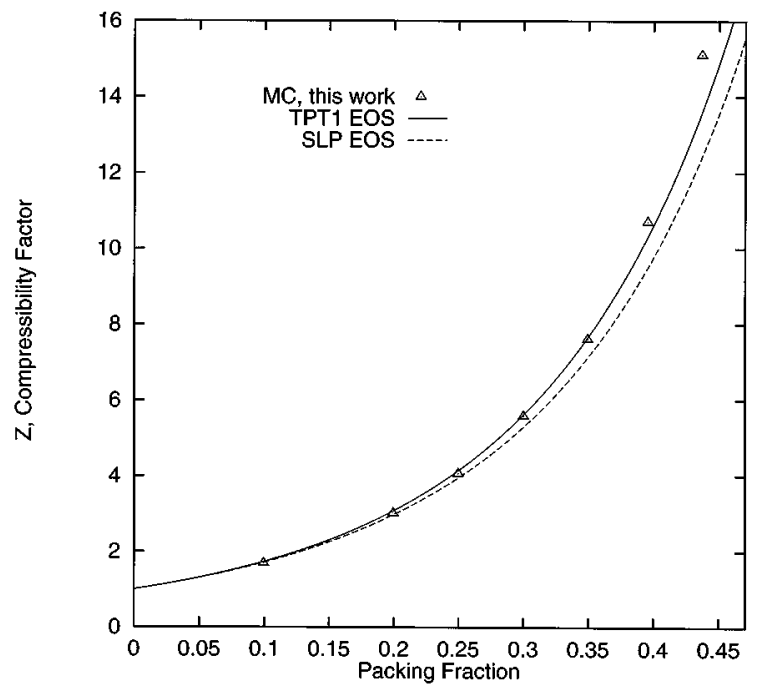

FIG. 18. Compressibility factor vs packing fraction for a hard-core mixture of 16-mer/monomer (50\% volume). Results of MC simulations of this work $(\triangle)$, the first-order thermodynamic perturbation theory (TPT1) (solid line) and the Song-Lambert-Prausnitz (SLP) (dashed line) equations of state. 


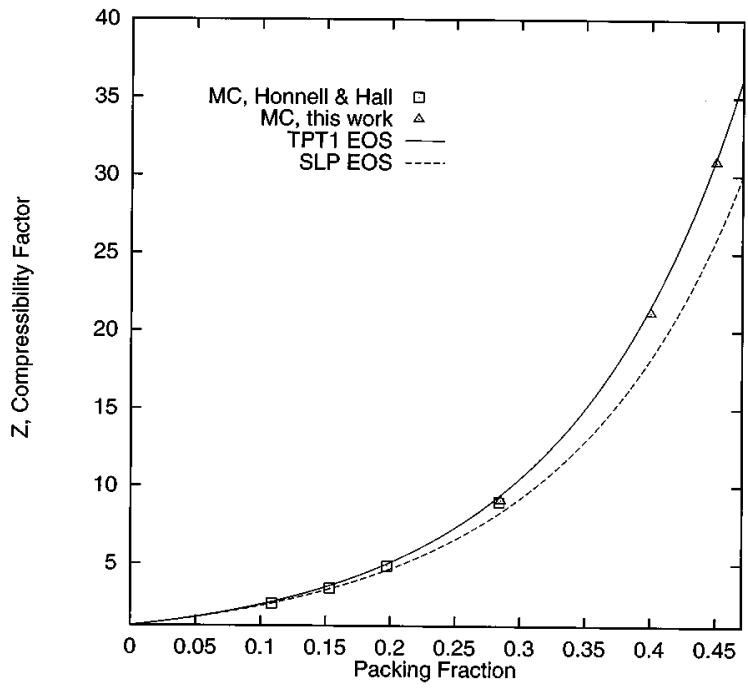

FIG. 19. Compressibility factor vs packing fraction for a hard-core mixture of 8 -mer/monomer ( $88.9 \%$ volume of chains). Results of MC simulations of Honnell and Hall (Ref. 13) $(\square)$ and this work $(\triangle)$. Predictions of the firstorder thermodynamic perturbation theory (TPT1) (solid line) and the SongLambert-Prausnitz (SLP) (dashed line) equations of state.

Wertheim ${ }^{17}$ ), and by Song et al. ${ }^{18}$ (based on Chiew's solution of the Ornstein-Zernicke equation for the associating fluid in the Percus-Yevick approximation ${ }^{19}$ ). On the one hand, it is observed in Figs. 16 and 17 that for the homopolymer systems, Chapman et al.'s equation tends to overestimate the compressibility factor for all but the highest density points. The deviations are, nevertheless, small. Song et al.'s equation, on the other hand, underestimates the compressibility factor. Note that the deviations from simulation tend to grow for higher packing fractions. Similar trends are observed for the binary systems (Figs. 18 and 19). The equation of Chapman et al. provides a good description of our 16-mer/ monomer simulation data for all but the highest densities, where the simulation points lie significantly above the theoretical curve. It is important to point out that the predictive capability of Song et al.'s equation was originally tested using the 8 -mer/monomer system of Fig. $19 .{ }^{18}$ However, only limited simulation data (in the medium-low density range ${ }^{13}$ ) were available at the time.

Chapman et al.'s equation and Song et al.'s equation have been already extended to deal with realistic systems. However, our results suggest that some improvements are still necessary at the hard-core-model level. In fact, several improved equations of state for hard-chains have already been proposed. ${ }^{20-22}$ However, a thorough testing of equationof-state performance should include hard core systems that exhibit a large asymmetry in the geometric characteristics of the molecules. As reported recently by Wichert and Hall, ${ }^{39}$ simulation data for asymmetric monomer/short-chain systems show larger deviations from theoretical predictions than symmetric systems.

\section{CONCLUSIONS AND FUTURE WORK}

The purpose of this paper has been to present new Monte Carlo methods for simulation of flexible molecules. The
ECCB proposed here is an extension of the original CCB method that enables the rearrangement of inner sites in linear or branched chains. Armed with this new tool, a means of producing localized, simultaneous rearrangements of sites from various chains has been presented (HOLE method). The results of NVT simulations for melts of hard-sphere chains show that the ECCB method clearly outperforms conventional methods (such as standard CCB and crank shaft) by producing faster molecular rearrangements (i.e., a faster decay of the bond orientation and end-to-end vector orientation autocorrelation functions). For simulation of polymer solutions, the HOLE method also provides substantial advantages over conventional techniques.

Our results for a homopolymer system of 51-mers and a binary system of monomer/16-mer provide the first reported exact PV data for such long chains. Results for the compressibility factor are consistent with previously reported simulation data (whenever available). However, some small deviations have been identified, particularly at high densities. The predictions of two well known equations of state have been compared to our simulation data; in spite of a generally satisfactory agreement, small systematic deviations occur at low and high densities.

Currently, more extensive simulation studies are under way for some systems for which the ECCB and HOLE methods are promising.

(i) Long homonuclear and heteronuclear chains at medium and high densities (pure systems and blends).

(ii) Ring polymers, branched chains, and crosslinked structures.

(iii) Semiflexible chains.

(iv) Simulation of chains in constrained environments such as pores.

A number of possible extensions of the methods presented here are also being considered. In particular, the HOLE method provides an excellent framework for the implementation of pseudokinetic moves, ${ }^{40}$ where chain sections of two chains are allowed to exchange identities. For instance, having identified within the hole two sets of sites that belong to two different but identical heteronuclear chains, an exchange of identity can be attempted if at least one equivalent site is present in the two sets (this condition can be relaxed for homonuclear chains).

An alternative method has also been developed to perform NPT-ensemble simulations (manuscript in preparation), where volume changes involve the creation of a HOLE of rectangular geometry (i.e., a "slice" of the simulation box). This slab is subsequently enlarged or reduced and the molecular segments contained in it are repositioned accordingly. This method would be advantageous for dense systems composed of a very large number of particles, where the chances of success for conventional 'global' volume moves ${ }^{23}$ become prohibitively small.

\section{ACKNOWLEDGMENTS}

The authors gratefully acknowledge the financial support of the National Science Foundation and from the industrial sponsors of the University of Wisconsin Polymerization Reaction Engineering Laboratory. One of us (J.J.dP.) is grateful 


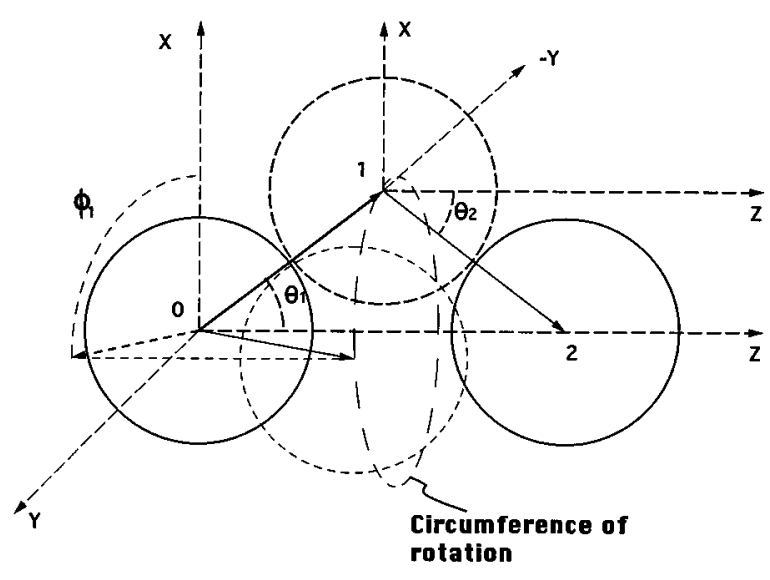

FIG. 20. Nomenclature and local coordinate system adopted for the transformation entailed by a crank-shaft move.

to the Camille and Henry Dreyfus Foundation for a new faculty award. Most of the calculations presented in this work were performed on an RS6000 workstation awarded to us by the IBM corporation.

\section{APPENDIX A: JACOBIAN OF THE CRANK-SHAFT TRANSFORMATION}

The crank-shaft move repositions a site by a restricted sampling of the configuration space. In the general case, a growing site is repositioned by specifying the two spherical angles of the linking bond vector, but a crank-shaft move requires the specification of only one of such angles. The nomenclature and coordinate system to be used are illustrated in Fig. 20. It is assumed that the chain is regrown starting from site 1 and up. Random values of $\phi_{i}$ (uniformly distributed in the $[0,2 \pi]$ interval) and $\cos \theta_{i}$ (distributed in the $[-1,1]$ interval) are used to fix the position of site $i$. A crank-shaft move can be envisioned as a regrowing process that, having started with site 1 , stops at site 2 . Then, the temporary change of degrees of freedom entailed by a simple rotation of site 1 around the $0-2$ axis is given by:

$$
\phi_{1}, \cos \theta_{1}, \phi_{2}, \cos \theta_{2} \rightarrow \phi_{1}, \mathbf{r}_{2},
$$

where $\mathbf{r}_{2}$ is the position vector of site 2 (which is fixed in the crank-shaft move). Since angle $\phi_{1}$ is a common degree of freedom, it can be removed from the transformation. The volume elements for both coordinate systems are related through the Jacobian determinant as

$$
\begin{aligned}
d\left(\cos \theta_{1}\right) d\left(\cos \theta_{2}\right) d \phi_{2} & =\left|\frac{\partial\left(\cos \theta_{1}, \cos \theta_{2}, \phi_{2}\right)}{\partial\left(r_{2_{x}}, r_{2_{y}}, r_{2_{z}}\right)}\right| d \mathbf{r}_{2} \\
& =\mathbf{J} d \mathbf{r}_{2} .
\end{aligned}
$$

The Jacobian determinant can be rewritten as

$$
\mathbf{J}=\sin \theta_{1} \sin \theta_{2}\left|\frac{\partial \mathbf{r}_{2}}{\partial \theta_{1}} \frac{\partial \mathbf{r}_{2}}{\partial \theta_{2}} \frac{\partial \mathbf{r}_{2}}{\partial \phi_{2}}\right|^{-1} \text {. }
$$

The position of site 2 can be expressed in terms of the spherical angles associated with the regrown sites as

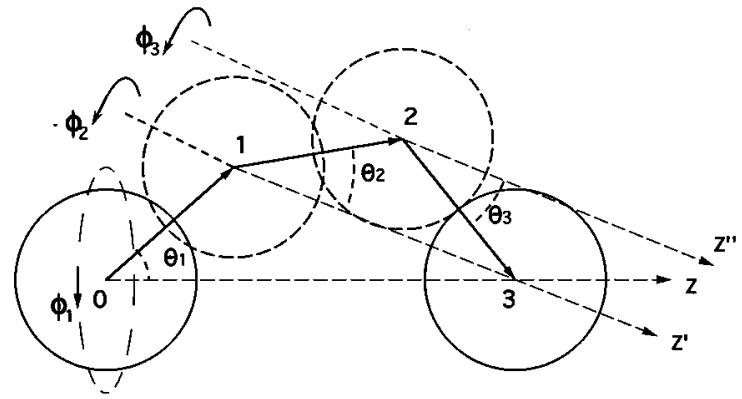

FIG. 21. Nomenclature and local coordinate system adopted for the transformation entailed by ECCB-type moves, here exemplified for the case of two growing sites (1 and 2).

$$
\begin{aligned}
\mathbf{r}_{2}= & \mathbf{r}_{0}+\sum_{i=1}^{2} b_{i-1, i}\left(\sin \theta_{i} \cos \phi_{i} \mathbf{u}_{x}+\sin \theta_{i} \sin \phi_{i} \mathbf{u}_{y}\right. \\
& \left.+\cos \theta_{i} \mathbf{u}_{z}\right),
\end{aligned}
$$

where $\mathbf{u}_{x}, \mathbf{u}_{y}$, and $\mathbf{u}_{z}$ are unit vectors along the corresponding axis of the temporary coordinate system. Differentiating Eq. (A4) we get

$\frac{\partial \mathbf{r}_{2}}{\partial \theta_{1}}=b_{0,1}\left(\cos \theta_{1} \cos \phi_{1} \mathbf{u}_{x}+\cos \theta_{1} \sin \phi_{1} \mathbf{u}_{y}-\sin \theta_{1} \mathbf{u}_{z}\right)$,

$\frac{\partial \mathbf{r}_{2}}{\partial \theta_{2}}=b_{1,2}\left(\cos \theta_{2} \cos \phi_{2} \mathbf{u}_{x}+\cos \theta_{2} \sin \phi_{2} \mathbf{u}_{y}-\sin \theta_{2} \mathbf{u}_{z}\right)$,

$\frac{\partial \mathbf{r}_{2}}{\partial \phi_{2}}=b_{1,2} \sin \theta_{2}\left(-\sin \theta_{2} \mathbf{u}_{x}+\cos \phi_{2} \mathbf{u}_{y}\right)$.

By construction of the coordinate system, the old configuration is specified by $\phi_{1}=0$ and $\phi_{2}=\pi$. It is easy to show that, for the new configuration (and for any value of $\phi_{1}$ chosen) $\phi_{2}=\phi_{1} \pm \pi$. Using these identities, and making all the necessary algebraic operations, Eq. (A3) reduces to

$$
\mathbf{J}=\frac{\sin \theta_{1}}{b_{0,1} b_{1,2}^{2} \sin \left(\theta_{1}+\theta_{2}\right)}=\frac{1}{b_{0,1} b_{1,2} d_{0,2}} .
$$

For the crank-shaft move, the distance $d_{0,2}$ is the same for the old and new configurations. Since our molecular model assumes fixed bond lengths, it follows that the Jacobian determinant will be the same for the old and new configurations and their ratio will always be unity.

\section{APPENDIX B: JACOBIANS OF THE ECCB TRANSFORMATION}

The transformation of degrees of freedom involved in a ECCB move will be first illustrated for the case when only 2 sites are rearranged. A generalized result will then be given. The framework for this move is displayed in Fig. 21. For the purpose of this derivation, the regrowing process occurs as if the three sites $(1,2$, and 3$)$ were to be relocated in a standard $\mathrm{CCB}$ fashion, with the restriction that site three has to be 
"relocated" in its original position. The local coordinate system for the ECCB move changes each time that a site is regrown, i.e., the $z$ axis is chosen to lie along the line that joins the centers of the two current open sites in the chain. This holds for all but the last site (3), in which case the $z$ axis is chosen parallel to the line 1-3. The directions of the $x$ and $y$ axis are arbitrary. Following similar arguments as those presented for the crank-shaft move, the transformation is

$$
\phi_{1}, \cos \theta_{1}, \phi_{2}, \cos \theta_{2}, \phi_{3}, \cos \theta_{3} \rightarrow \phi_{1}, \phi_{2}, \cos \theta_{1}^{*}, \mathbf{r}_{3} .
$$

The superscript $*$ denotes that the variable has a restricted range of values. Removing the common degrees of freedom $\phi_{1}$ and $\phi_{2}$ from the transformation, the volume elements in the two coordinate frames are related by

$$
d\left(\cos \theta_{1}\right) d\left(\cos \theta_{2}\right) d\left(\cos \theta_{3}\right) d \phi_{3}=\mathbf{J} d \mathbf{r}_{3} d\left(\cos \theta_{1}^{*}\right),
$$

where

$$
\begin{aligned}
\mathbf{J}^{-1} & =\left|\frac{\partial\left(\cos \theta_{1}^{*}, \mathbf{r}_{3}\right)}{\partial\left(\cos \theta_{1}, \cos \theta_{2}, \cos \theta_{3}, \phi_{3}\right)}\right| \\
& =\left|\begin{array}{llll}
\frac{\partial \cos \theta_{1}^{*}}{\partial \cos \theta_{1}} & \frac{\partial \cos \theta_{1}^{*}}{\partial \cos \theta_{2}} & \frac{\partial \cos \theta_{1}^{*}}{\partial \cos \theta_{3}} & \frac{\partial \cos \theta_{1}^{*}}{\partial \phi_{3}} \\
\frac{\partial \mathbf{r}_{3}}{\partial \cos \theta_{1}} & \frac{\partial \mathbf{r}_{3}}{\partial \cos \theta_{2}} & \frac{\partial \mathbf{r}_{3}}{\partial \cos \theta_{3}} & \frac{\partial \mathbf{r}_{3}}{\partial \phi_{3}}
\end{array}\right| .
\end{aligned}
$$

Note that, in general, $\partial \cos \theta_{1}^{*} / \partial \phi_{3}=0$. If the new position of site 1 has been generated by mode-2 sampling, i.e., by choosing $\cos \theta_{1}$ randomly and uniformly over the range $\left[\cos \theta_{1_{\max }}, \cos \theta_{1_{\text {min }}}\right]$, then the partial derivatives $\partial \cos \theta_{1}^{*} / \partial \cos \theta_{i}(i=1,2)$ will not be trivial to evaluate since they are functions of the bounds of $\cos \theta_{1}$. However, using mode-1 sampling, $\cos \theta_{1}^{*}=\cos \cos \operatorname{coscos} \theta_{1}$ and thus the Jacobian determinant reduces to

$$
\begin{aligned}
\mathbf{J}^{\text {mode } 1} & =\left|\frac{\partial\left(\mathbf{r}_{3}\right)}{\partial\left(\cos \theta_{2}, \cos \theta_{3}, \phi_{3}\right)}\right|^{-1} \\
& =\sin \theta_{2} \sin \theta_{3}\left|\frac{\partial \mathbf{r}_{3}}{\partial \theta_{2}} \frac{\partial \mathbf{r}_{3}}{\partial \theta_{3}} \frac{\partial \mathbf{r}_{3}}{\partial \phi_{3}}\right|^{-1} .
\end{aligned}
$$

This expression is completely equivalent to Eq. (A3) for the crank-shaft move and the final result is analogous:

$$
\mathbf{J}^{\text {mode } 1}=\frac{\sin \theta_{2}}{b_{1,2} b_{2,3}^{2} \sin \left(\theta_{2}+\theta_{3}\right)}=\frac{1}{b_{1,2} b_{2,3} d_{1,3}} .
$$

The general result for mode-1 sampling when $k-1$ sites have been cut, $k-1$ being the last site to be regrown, is simply

$$
\mathbf{J}^{\text {mode } 1}=\frac{1}{b_{k-2, k-1} b_{k-1, k} d_{k-2, k}} .
$$

The critical difference from the crank-shaft result is that now $\mathbf{J}$ is not the same for the old and new configurations, since $d_{k-2, k}$ is constant only if $k=2$ (crank-shaft case).

The Jacobian determinant for mode-2 sampling can be obtained in a number of ways. The more straightforward approach is to simply argue that the volume element associated with this sampling is a fraction of that corresponding to mode-1 sampling. This fraction is the ratio of the spherical sector area sampled by mode- $\mathbf{2}$ sampling divided by the total surface of the sphere (which is sampled by mode-1 sampling), thus

$$
\begin{aligned}
& \mathbf{J}^{\text {mode } 2}=\frac{\text { spherical sector sampled }}{\text { total area sphere }} \mathbf{J}^{\text {mode } 1,} \\
& \mathbf{J}^{\text {mode } 2}=\frac{1}{2}\left(\cos \theta_{1_{\text {min }}}-\cos \theta_{1_{\text {max }}}\right) \frac{1}{b_{1,2} b_{2,3} d_{1,3}} .
\end{aligned}
$$

The general result for $k-1$ regrown sites is

$\mathbf{J}^{\text {mode } 2}=\frac{(1 / 2)^{k-2}}{b_{k-2, k-1} b_{k-1, k} d_{k-2, k}} \prod_{i=1}^{k-2}\left(\cos \theta_{i_{\min }}-\cos \theta_{i_{\max }}\right)$.

For the HOLE method, the mode-1 sampling Jacobian determinant for the whole move will be given by a square matrix of $3 \times N_{\text {sets }}$ rows, where $N_{\text {sets }}$ is the number of sets of sites cut in the hole. Many of the entries in such a matrix will be zero, because the partial derivative of a degree of freedom from one set of sites with respect to a degree of freedom from a different set is zero. It can be shown by means of the Laplace expansion of determinants that the total determinant can be expressed as

$$
\mathbf{J}_{\mathrm{HOLE}}^{\text {mode } 1}=\prod_{j=1}^{N_{\text {sets }}} \mathbf{J}_{j}^{\text {mode } 1}
$$

where $\mathbf{J}_{j}^{\text {mode } 1}$ is the Jacobian determinant corresponding to the $j$ th set of sites and is given by an expression analogous to Eq. (B6).

Similar arguments can be applied when mode-2 sampling is adopted in the context of the HOLE method.

For the case of rearranging sites that include a branchlinking site, the Jacobian determinant consistent with mode-1 sampling developed in Sec. II D can be evaluated by analogy to the previous cases. During the first step of the regrowing process (from one of the open sites up to the linking site), only an "energetic" constraint is enforced to ensure that no regrown site is placed out of range of the maximal extension of the connecting bonds along the "threads" to be closed. In the second step of the process (from the linking site to remaining open sites), the two "threads" are regrown independently, i.e., as two distinct sets of sites in analogy to the HOLE method. Furthermore, no change or restriction in the degrees of freedom is inherited from the the first step of the process. It follows that the Jacobian determinant for this case is given by

$$
\mathbf{J}_{\text {branch }}^{\text {mode } 1}=\mathbf{J}_{\text {thread }{ }_{1}}^{\text {mode } 1} \mathbf{J}_{\text {thread 2 }}^{\text {mode } 1}
$$

where $\mathbf{J}_{\text {thread } i}^{\text {mode }}{ }_{i}$ is given by Eq. (B6) once the proper $k$ value is used, consistent with the sites cut in the $i$ th thread.

A special case arises when the branch-linking site is the last to be regrown; its relocation has no degrees of freedom and therefore no Jacobian associated to it. 
${ }^{1}$ M. Bishop, M. H. Kalos, and H. L. Frisch, J. Chem. Phys. 70, 1299 (1979); M. Bishop, D. Ceperley, H. L. Frisch, and M. H. Kalos, ibid. 72, 3228 (1980).

${ }^{2}$ K. Kremer and G. Grest, J. Chem. Phys. 92, 5057 (1990).

${ }^{3}$ Applications of the Monte Carlo Method in Statistical Physics, edited by K. Binder (Springer, New York, 1987).

${ }^{4}$ Computational Modeling of Polymers, edited by $\mathrm{K}$. Binder and $\mathrm{J}$. Bicerano (Marcel-Dekker, New York, 1992).

${ }^{5}$ J. I. Siepmann and D. Frenkel, Mol. Phys. 75, 59 (1992).

${ }^{6}$ J. J. de Pablo, M. Laso, and U. W. Suter, J. Chem. Phys. 96, 2395 (1992).

${ }^{7}$ J. J. de Pablo, M. Laso, and U. W. Suter, J. Chem. Phys. 96, 6157 (1992).

${ }^{8}$ L. R. Dodd, T. D. Boone, and D. N. Theodorou, Mol. Phys. 78, 961 (1993).

${ }^{9}$ E. Leontidis, J. J. de Pablo, M. Laso, and U. W. Suter, Advances in Polymer Science (Springer-Verlag, New York, 1994), Vol. 116, p. 285.

${ }^{10}$ J. P. Hansen and I. R. MacDonald, Theory of Simple Liquids (Academic, New York, 1986).

${ }^{11}$ A. Yethiraj and C. K. Hall, J. Chem. Phys. 96, 797 (1992).

${ }^{12}$ K. G. Honnell and C. K. Hall, J. Chem. Phys. 90, 1841 (1989).

${ }^{13}$ K. G. Honnell and C. K. Hall, J. Chem. Phys. 95, 4481 (1991).

${ }^{14}$ A. Yethiraj and C. K. Hall, Mol. Phys. 80, 469 (1993).

${ }^{15}$ R. Dickman and C. K. Hall, J. Chem. Phys. 89, 3168 (1988).

${ }^{16}$ W. G. Chapman, G. Jackson, and K. E. Gubbins, Molec. Phys. 65, 1057 (1988).

${ }^{17}$ M. S. Whertheim, J. Chem. Phys. 87, 7323 (1987).

${ }^{18}$ Y. Song, M. Lambert, and J. M. Prausnitz, Macromol. 27, 441 (1994).

${ }^{19}$ Y. C. Chiew, Mol. Phys. 70, 129 (1990).

${ }^{20}$ S. Phan, E. Lierlik, M. L. Rosinberg, H. Yu, and G. Stell, J. Chem. Phys. 99, 5326 (1993).
${ }^{21}$ J. Chang and S. I. Sandler, Chem. Eng. Sci. 49, 2777 (1994).

${ }^{22}$ D. Ghonasgi and W. G. Chapman, J. Chem. Phys. 100, 6633 (1994).

${ }^{23}$ M. P. Allen and D. J. Tildesley, Computer Simulation of Liquids (Clarendon, Oxford, 1987).

${ }^{24}$ J. Gao and J. H. Weiner, J. Chem. Phys. 91, 3168 (1989).

${ }^{25}$ M. Dijkstra, D. Frenkel, and J. P. Hansen, J. Chem. Phys. 101, 3179 (1994).

${ }^{26}$ R. H. Boyd, Macromolecules 22, 2477 (1989).

${ }^{27}$ N. G. Almarza, E. Enciso, and F. J. Bermejo, J. Chem. Phys. 96, 4625 (1992).

${ }^{28}$ B. M. Forrest and U. W. Suter, J. Chem. Phys. 101, 2616 (1994).

${ }^{29}$ X. Li and Y. C. Chiew, J. Chem. Phys. 101, 2522 (1994).

${ }^{30}$ S. K. Kumar, M. Vacatello, and D. Y. Yoon, J. Chem. Phys. 89, 5206 (1988).

${ }^{31}$ P. H. Verdier and W. H. Stockmayer, J. Chem. Phys. 36, 227 (1962).

${ }^{32}$ H. A. Kramers, Physica 11, 1 (1944).

${ }^{33}$ M. N. Rosenbluth and A. W. Rosenbluth, J. Chem. Phys. 23, 356 (1955).

${ }^{34}$ H. Meirovich, Phys. Rev. A 32, 3699 (1985).

${ }^{35}$ S. Livne and H. Meirovich, J. Chem. Phys. 88, 4498 (1988).

${ }^{36}$ J. I. Siepmann, Mol. Phys. 70, 1145 (1990).

${ }^{37}$ D. Frenkel, G. C. A. M. Mooij, and B. Smit, J. Phys. Condens. Matt, 4, 3053 (1992).

${ }^{38} \mathrm{~S}$. W. Smith (unpublished), as referenced by Yethiraj and Hall (Ref. 14).

${ }^{39}$ J. M. Wichert and C. K. Hall, Chem. Eng. Sci. 49, 2793 (1994).

${ }^{40}$ M. Lastoskie and W. G. Madden, Computer Simulation of Polymer, edited by R. J. Roe (Prentice-Hall, New York, 1991), p. 233. 